\title{
Node Sampling for Nonlinear Vibration Analysis of Structures with Intermittent Contact
}

\author{
Akira Saito* and Bogdan I. Epureanu \\ University of Michigan, Ann Arbor, Michigan 48109-2125 \\ Matthew P. Castanier \\ U.S. Army Tank Automotive Research, Development, and Engineering Center, \\ Warren, Michigan 48397-5000 \\ and \\ Christophe Pierre $\underline{\S}$ \\ McGill University, Montreal, Quebec H3A 2K6, Canada \\ DOI: $\underline{10.2514 / 1 . J 050061}$
}

\begin{abstract}
In this paper, a node sampling methodology for nonlinear vibration problems of elastic structures involving intermittent contact is proposed. Of particular interest is a vibration problem of platelike elastic structures with a crack, with a large number of degrees of freedom involved on the crack surfaces. Because of the localized nature of such nonlinearity, the number of degrees of freedom on the surfaces greatly affects the computational time of the analysis. Therefore, reducing the number of degrees of freedom on the crack surfaces without significantly sacrificing the accuracy of the results is a critical issue for conducting vibration analysis of such structures in a reasonable amount of time. The focus is placed on the development of an efficient algorithm to select a set of nodes on the crack surfaces, where nonlinear boundary conditions are imposed. The method is developed, based on a procedure for selecting master degrees of freedom for Guyan reduction. The accuracy, efficiency, and optimality of the method are discussed in detail and compared with those aspects of previous methods. The advantages of the new method are demonstrated in terms of the accuracy of the frequency response and the resonant frequencies.
\end{abstract}

\begin{tabular}{|c|c|c|}
\hline \multicolumn{3}{|r|}{ Nomenclature } \\
\hline$A$ & $=$ & assembly operator \\
\hline $\mathcal{B}$ & $=$ & $\begin{array}{l}\text { set of degree-of-freedom indices corresponding to } \\
\text { boundary nodes }\end{array}$ \\
\hline $\mathcal{C}_{c p}$ & $=$ & $\begin{array}{l}\text { set of degree-of-freedom indices corresponding } \\
\text { the contact pairs }\end{array}$ \\
\hline $\mathcal{D}$ & $=$ & $\begin{array}{l}\text { set of degree-of-freedom indices corresponding to } \\
\text { deleted degrees of freedom }\end{array}$ \\
\hline$\hat{F}$ & $=$ & $\begin{array}{l}\text { virtual impulse associated with the amount of } \\
\text { penetration }\end{array}$ \\
\hline $\mathbf{f}$ & $=$ & contact force vector \\
\hline $\mathcal{G}$ & $=$ & $\begin{array}{l}\text { set of degrees of freedom corresponding to all } \\
\text { generalized internal degrees of freedom }\end{array}$ \\
\hline $\mathbf{H}$ & $=$ & $\begin{array}{l}\text { coordinate transformation matrix of the } \\
\text { Hintz-Herting component mode synthesis method }\end{array}$ \\
\hline I & $=$ & identity matrix \\
\hline $\mathcal{I}$ & $=$ & $\begin{array}{l}\text { set of degree-of-freedom indices corresponding to } \\
\text { internal nodes }\end{array}$ \\
\hline$k_{e}$ & $=$ & $\begin{array}{l}\text { equivalent spring constant per unit length on crack } \\
\text { faces }\end{array}$ \\
\hline
\end{tabular}

Presented as Paper 2009-2493 at the 50th AIAA/ASME/ASCE/AHS/ASC Structures, Structural Dynamics, and Materials Conference, Palm Springs, CA, 4-7 May 2009; received 14 July 2009; revision received 12 May 2010; accepted for publication 18 May 2010. Copyright (C) 2010 by Akira Saito, Bogdan I. Epureanu, Matthew P. Castanier, and Christophe Pierre. Published by the American Institute of Aeronautics and Astronautics, Inc., with permission. Copies of this paper may be made for personal or internal use, on condition that the copier pay the $\$ 10.00$ per-copy fee to the Copyright Clearance Center, Inc., 222 Rosewood Drive, Danvers, MA 01923; include the code 0001-1452/10 and $\$ 10.00$ in correspondence with the CCC.

*Graduate Student Research Assistant, Department of Mechanical Engineering; currently Assistant Researcher, Toyota Central R\&D Labs, Inc., Vehicle Mechanism Laboratory, Vehicle and Biomechanics Systems Division, Nagakute, Aichi 480-1192, Japan. Student Member AIAA.

${ }^{\dagger}$ Associate Professor, Department of Mechanical Engineering. Member AIAA.

${ }^{\ddagger}$ Mechanical Engineer, Research Business Group.

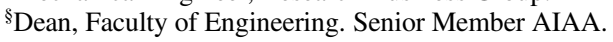

$\mathcal{L}$

$\mathcal{M}$

$\mathbf{M}, \mathbf{K}$

$\mathbb{M}, \mathbb{K}$

$\mathbf{M}_{H}, \mathbf{K}_{H}=$ finite-element mass and stiffness matrices

$\mathcal{N}$

$n$

$n_{m}$

$\mathbf{n}_{1}^{j}, \mathbf{n}_{2}^{j}, \mathbf{n}_{3}^{j}$

$\mathcal{O}$

$\mathbf{P}^{j}, \mathbf{P}$

$\stackrel{\mathbf{q}}{\mathcal{R}}$

$\mathbf{R}^{j}, \mathbf{R}$

$T$

$u$

$u^{j}, v^{j}$

$u_{p}$

$\mathcal{X}, \mathcal{Y}, \mathcal{Z}=\begin{aligned} & \text { on crack surface } \\ & \text { set of degree-of-freedom indices corresponding to }\end{aligned}$

$x_{1}, x_{2}$, and $x_{3}$

$\mathbf{x}=$ finite-element nodal displacement vector

$x_{1}, x_{2}, x_{3}=$ perpendicular axes of Cartesian coordinate system

$\Gamma_{A, B}=$ boundaries involving intermittent contact 


$\begin{array}{lll}\Gamma_{f} & = & \text { fixed boundary } \\ \eta & = & \text { modal coordinates corresponding to } \mathbf{H} \\ \boldsymbol{\Phi}, \hat{\boldsymbol{\Phi}} & = & \text { matrix of free-interface normal modes and its } \\ & & \text { modified form } \\ \boldsymbol{\phi} & = & \text { eigenvector of the finite-element model } \\ \boldsymbol{\Psi} & = & \text { matrix of constraint modes } \\ \Omega & = & \text { domain occupied by the elastic structure } \\ \omega_{b i} & = & \text { bilinear frequency } \\ \omega_{o} & = & \text { natural frequency with the open boundary } \\ & = & \text { condition } \\ \omega_{s} & = & \text { natural frequency with the sliding boundary } \\ & & \text { condition }\end{array}$

Superscript

$j \quad=$ contact pair

Subscripts

$\begin{array}{lll}A, B & = & \text { boundary degrees of freedom corresponding to } \\ & & \Gamma_{A} \text { or } \Gamma_{B} \\ a & = & \text { active degrees of freedom } \\ b & = & \text { boundary degrees of freedom } \\ d & = & \text { deleted degrees of freedom } \\ g & = & \text { generalized internal degrees of freedom } \\ i & = & \text { internal degrees of freedom } \\ l & = & \text { degrees of freedom not exposed to nonlinearity } \\ m & = & \text { modal coordinates } \\ r & = & \text { relative degrees of freedom }\end{array}$

\section{Introduction}

$\mathbf{V}$ IBRATION problems of structures with intermittent contact have been studied extensively for several decades. These problems have practical importance and feature theoretical complexity due to their nonlinear nature. A numerical modeling procedure for such problems, based on the finite-element (FE) method, is presented in this paper. This work is motivated by a need for developing a model-based crack detection algorithm of elastic structures, based on their spectral properties, such as resonant frequencies and response shapes. To properly predict the resonant frequencies of such structures, one has to consider the nonlinearity caused by intermittent contact at the cracks; the so-called closing crack or breathing crack effect. This nonlinearity has hindered analysts from accurately calculating the resonant frequencies of cracked structures, because they cannot be calculated from classical linear modal analysis. Recently, sophisticated contact algorithms have been developed, such as the penalty method [1] and the augmented Lagrangian method [2]. Hence, the accuracy of the results of time transient simulations with FE models involving intermittent contact has been improved. Furthermore, studying vibration problems of such structures with an FE model with a realistic complexity is becoming feasible with the aid of high-performance computers. However, in turn, due to the advancement of these technologies, analysts tend to create models with increasingly larger numbers of degrees of freedom (DOFs). This is based on the expectation that, as the models become more realistic and the results become more accurate, the problem can still be solved in a reasonable amount of time. However, the number of DOFs required for highfidelity predictions currently overwhelms even the most advanced hardware and software. That is because, as the model complexity increases, the cost of solving contact problems increases dramatically, even when the potential contact areas are known a priori. This occurs even if one uses reduced-order modeling techniques, such as the Craig-Bampton method [3]. For forced response vibration problems of such structures, one can use accurate and efficient semianalytical methods, such as the ones based on the harmonic balance method (e.g., [4]), by representing the steady-state dynamic response of the model with a truncated Fourier series. However, such methods still suffer from the increase of computational cost, as they require a fair number of harmonics to be included in the Fourier transform, to obtain an accurate result. Therefore, the goal of this paper is to present a new and efficient reduced-order modeling framework for vibration problems of elastic structures involving intermittent contact, with particular attention to modeling nonlinear vibration of cracked structures. The reduced-order model is constructed such that it can be used in conjunction with standard contact algorithms, such as Lagrange's multipliers, penalty methods, and augmented Lagrangian methods. The focus is placed upon reducing the number of DOFs involved in the contact regions, in an automatic manner.

This paper is organized as follows. In Sec. II, a literature survey over the related fields is provided. In Sec. III, the proposed modeling framework is presented, including the reduced-order modeling approach and contact DOFs selection method. As applications of the method, two case studies are shown in Sec. IV, using FE models of a cantilevered cracked plate and an academic blade model. Conclusions of the paper are then given in Sec. $\underline{V}$.

\section{Background}

The issues of reducing and selecting DOFs of FE models have been extensively studied by various methods and distinct perspectives, such as the reduction of the interface DOFs between substructures, the selection of master DOFs for Guyan reduction [5], the optimal sensor placement, and the optimal constraint locations. However, many of the available methods share similar goals and relate to each other (as described next).

First, the issue of reducing the number of interface DOFs between the components has been studied by several researchers. Brahmi et al. [6] proposed a method to be employed before the assembly of substructures in component-based modeling methods, where basis vectors are chosen based on the combination of secondary modal analysis of the interface DOFs partitions of the matrices and the truncation of modes based on the singular value decomposition. Balmés [7] introduced the framework for generalizing interface DOFs, such as constraint modes, by considering the new basis representing the actual interface displacements. Castanier et al. [8] also proposed a technique based on applying modal analysis and mode truncation to the constraint mode partition of the matrices produced by component mode synthesis (CMS) [3] . The resulting modes are transformed back into the FE coordinates and are called characteristic constraint modes. All of these methods achieve a reduction in the number of interface DOFs. However, they do not provide any criteria as to how the interface DOFs need be selected automatically (or even manually) for accurately enforcing the boundary conditions (BCs) in the areas of intermittent contact.

Second, the selection of master DOFs is a crucial factor for determining the spectral properties of reduced-order models obtained by using Guyan reduction [5]. Hence, many algorithms for the selection of the master DOFs have been developed. These methods are relevant to our objective, because they produce results that often solve the optimization problem considered in this study (as discussed in Sec. III). An automatic master DOF selection algorithm was first proposed by Henshell and Ong [9] , in which the master DOFs are chosen where the inertia is high and the stiffness is low, whereas the slave DOFs are chosen where the inertia is low and the stiffness is high. This process can be automated by examining the radian frequency $\omega_{s}$ defined by fixing all DOFs except the DOF index $s$. Namely, $\omega_{s} \triangleq \sqrt{k_{s s} / m_{s s}}$, for $s=1, \ldots, n$, where $k_{i j}$ and $m_{i j}$ are the entries at the $i$ th row and $j$ th column in FE stiffness and mass matrices of size $n$. The index $s$ with the largest $\omega_{s}$ is identified at each iteration step, and the DOF is eliminated by an application of Guyan reduction [5], with $s$ being the slave DOFs and all the other DOFs being the master DOFs. This process can be repeated until the number of master DOFs reaches the desired magnitude. An approach similar to this algorithm was proposed by Shah and Raymund [10], based on the discussions of Kidder [11,12] and Flax [13], where the number of master DOFs is controlled by iteratively eliminating the DOFs for which the $\omega_{s}$ is larger than a predefined cutoff frequency $\omega_{c}$ (that is chosen to be approximately three times the highest significant frequency in the frequency range of interest). Independently from the work by Henshell and Ong [9], Grinenko and Mokeev [14] 
developed an order-reduction technique, named frequency dynamic condensation, which also uses a criterion to select master DOFs. Although their criterion was legitimate, the implementation of the selection algorithm still suffers from tedious exhaustive-search calculations for selecting the master DOFs. The selection method proposed by Matta [15] also uses the ratio $k_{s s} / m_{s s}$ with a criterion similar to that proposed by Henshell and Ong [9]. This method can be applied to not only Guyan reduction [5] but also to CMS, where both static and normal modes are used as basis vectors, onto which the system dynamics are projected. A method proposed by Bouhaddi and Fillod [16] uses a different concept where, if a DOF $a$ is a node of the $i$ th eigenmode, then fixing the DOF $a$ results in $\hat{\lambda}_{i-1}=\lambda_{i}$, where $\lambda_{i}$ is the $i$ th eigenvalue of the nonfixed system, and $\hat{\lambda}_{i-1}$ is the eigenvalue of the system with the DOFs $a$ fixed. This concept may be understood, using a vibration problem of a string with both ends fixed. That is, the lowest natural frequency of the string with a single support becomes the highest if the support is placed at the node of the second mode of vibration [17]. This is because the first mode with the constraint becomes identical to the second mode of the unconstrained string, which has the eigenvalue as the feasible upper bound of the first eigenvalue with a single constraint. It is noteworthy that Bouhaddi and Fillod [16] explicitly aimed for maximizing the minimum eigenvalue of the system, where all the master DOFs are fixed. This concept will be revisited in Sec. III.C.

The methods for the node selection reviewed previously are based on the Henshell and Ong [9] method to some extent. Another class of methods is based on the concept of modal energy. For example, the method proposed by Kim and Choi [18] uses the energy distribution among the DOFs for each mode, and it chooses the set of primary DOFs by taking the partial sum over the rows of the energy distribution matrix. The method proposed by Cho and Kim [19] uses energy estimation at the discretization element level by the Rayleigh quotient value of each element. Kim and Cho [20] then proposed a selection method consisting of two steps: model order reduction by improved reduced system (IRS) [21], using the master DOFs selected via the method based on energy estimation of each element [19], and the subsequent sequential elimination method [9], with an iterative IRS. Another automatic DOF selection method, named the modal energy selection method, proposed by $\mathrm{Li}$ [22], uses a metric called index of classification, which is based on the approximate modal energy associated with each DOF. The method was successfully applied to an FE model of a cantilever beam. Oh and Park [23] also proposed a criterion for selecting the master DOFs, based on singular values of the modal matrix. However, that approach suffers from the computational cost due to exhaustive search over the possible master DOF sets, and depends on engineer knowledge and intuition.

Third, a similar but slightly different issue is the selection of measurement locations for vibration testing. For example, one may need to measure vibration displacements of a structure to determine vibration modes, typically with a limited number of sensors and limited locations where the sensors can be placed. Thus, one may like to maximize the information one can obtain with the limited number of sensors and locations. However, the question arises as to how the sensors need to be located, since the optimal configuration of sensors for such objectives cannot be easily determined. There have been many methods developed to date for achieving this goal. In particular, one class of approach is based on information theory. These approaches determine the sensor locations by optimizing the norm of the Fisher information matrix [24]. Among them, one of the most widely used techniques is the effective independence vector method, or the EIDV [25] method, developed by Kammer [26]. The method determines the placement of sensors within the candidate locations while maintaining as much independent information as possible (i.e., maintaining the measured mode shapes as independent as possible). Therefore, it is natural to hypothesize that the application of the nonlinear BCs to the optimum sensor locations would also well represent the full-order model, where the BCs are applied at all locations in the intermittent contact region. This method is considered in this study, and the associated formulation is discussed in detail in Sec. III.C.
Last, the issue of finding the optimal constraint locations to maximize the fundamental natural frequency of a structure is considered. This issue has an important relationship with the optimal master DOF selection. For instance, suppose one wants to increase the lowest natural frequency of a structure as much as possible by adding a finite number of supports or kinematical constraints to the structure. However, the problem of finding the optimal number and the locations of such supports is not as easy as it appears. Therefore, it may be necessary to apply computationally expensive optimization algorithms, such as done in the work by Zhu and Zhang [27]. In contrast, Åkesson and Olhoff [28] studied the problem by applying the Courant's maximum-minimum principle. Namely, if there is a discrete dynamical system of size $n$, and there are $r(<n)$ kinematical constraints applied to the system, then all the eigenvalues of the structure increase, and the increased eigenvalues are bounded by the following formula:

$$
\lambda_{i}^{0} \leqslant \lambda_{i} \leqslant \lambda_{i+r}^{0}, \quad i \in\{1,2, \ldots, n\}
$$

where $\lambda_{i}^{0}$ and $\lambda_{i+r}^{0}$ denote the $i$ th and $(i+r)$ th eigenvalues of the structure without the constraints, and $\lambda_{i}$ is the $i$ th eigenvalue of the constrained structure. Also, based on the same principle and the findings of Szelag and Mroz [29], Won and Park [30] applied a minimization method to obtain the optimal support location and achieve the maximum fundamental natural frequency of a cantilevered plate. They showed that the optimal support locations should be on the nodal lines of the $(r+1)$ th mode of the unconstrained structure. It is noted that this result conforms to the vibration problem of a fixed string, mentioned previously. This method was successfully applied to their specific examples, but the method can be applied only to special cases where the potentially constrained region is the entire region of the structure and the points on the nodal lines can be selected. Namely, if the regions to which the constraints are applied are limited to some specific regions of the structure, then the nodal lines may not exist in such regions, and the minimization problem becomes more complicated.

It is interesting to note that the idea of constraining the nodal lines was used to optimally select the master DOFs for Guyan reduction [5] by Bouhaddi and Fillod [16], but they were not aware of the applicability of their method to optimally select the support positions, while Won and Park [30] were not aware of the applicability of their method to optimally select the master DOF locations for Guyan reduction [5]. In this paper, we take advantage of this similarity between the optimal master DOF selections and the constraint locations in order to achieve the optimal selection of the DOFs where the nonlinear BCs are applied.

\section{Mathematical Formulation}

Consider the small vibrations of an elastic structure, represented as $\Omega$, with a fixed boundary $\Gamma_{f}$, where the structure may involve intermittent contact at $\Gamma_{A}$ and $\Gamma_{B}$ during the vibration cycles, such as shown in Fig. 1. Namely, the boundaries open and close. Thus, the vibration problem is nonlinear, because the condition for the boundaries to be in contact is dependent on the displacement field

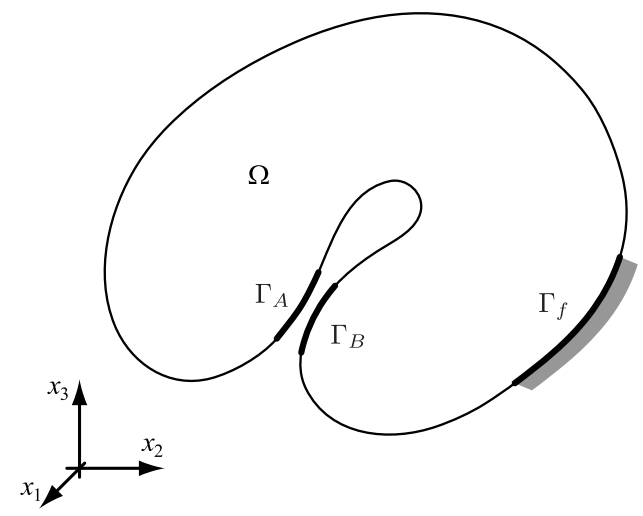

Fig. 1 An elastic structure with potentially contacting boundaries. 
itself. That is, the $\mathrm{BCs}$ at $\Gamma_{A}$ and $\Gamma_{B}$ are nonlinear. It is well known that the system eigenvectors and eigenfrequencies are different from the actual response shapes and resonant frequencies of this nonlinear structure. In this paper, they are, respectively, referred to as the nonlinear normal modes (NNMs) and NNM frequencies, as was also done in [31].

If the structure is discretized with a method such as an FE method, the nonlinearity associated with the contact is localized, in the sense that the nonlinearity is caused only by a small portion of the entire structure. In the following formulations, a set of indices of DOFs in such region is denoted as $\mathcal{B}$ (boundary), whereas a set of indices of the DOFs in the rest of the regions is denoted as $\mathcal{I}$ (internal), and partitions of vectors and matrices associated with these sets are designated with subscripts $b$ and $i$. The sizes of the sets are denoted as $|\mathcal{B}|=n_{\mathcal{B}}$ and $|\mathcal{I}|=n_{\mathcal{I}}$. All the other DOF sets defined hereinafter follow the same notation.

Consider that the FE mass and stiffness matrices are denoted by $\mathbf{M} \in \mathbb{R}^{n \times n}$ and $\mathbf{K} \in \mathbb{R}^{n \times n}$, and the nodal displacement vector is given by $\mathbf{x} \in \mathbb{R}^{n}$. The governing equations of the vibration problem, with the absence of external forcing and damping, may be written in a partitioned matrix-vector form as follows:

$$
\left(\begin{array}{ll}
\mathbf{M}_{b b} & \mathbf{M}_{b i} \\
\mathbf{M}_{i b} & \mathbf{M}_{i i}
\end{array}\right)\left[\begin{array}{c}
\ddot{\mathbf{x}}_{b} \\
\ddot{\mathbf{x}}_{i}
\end{array}\right]+\left(\begin{array}{ll}
\mathbf{K}_{b b} & \mathbf{K}_{b i} \\
\mathbf{K}_{i b} & \mathbf{K}_{i i}
\end{array}\right)\left[\begin{array}{c}
\mathbf{x}_{b} \\
\mathbf{x}_{i}
\end{array}\right]=\left[\begin{array}{c}
\mathbf{f}_{b}\left(\mathbf{x}_{b}\right) \\
\mathbf{0}
\end{array}\right]
$$

where () denotes a time derivative, and $\mathbf{f}_{b} \in \mathbb{R}^{n_{\mathcal{B}}}$ denotes the nonlinear force associated with the intermittent contact.

When dealing with this type of nonlinear vibration problems, one can apply linear reduced-order modeling techniques, such as Guyan reduction [5], system equivalent reduction expansion process [32], iterated IRS $[33,34]$, or component mode synthesis (CMS) [3]. With such methods, one can obtain smaller system matrices by reducing the size of $\mathbf{x}_{i}$ by means of Rayleigh-Ritz coordinate transformation, comprising of various basis vectors such as static deformations and vibration modes yet keeping the accessibility to the physical coordinates of $\mathbf{x}_{b}$. For instance, with the help of CMS, one can obtain a system with desired spectral properties and accessibility to $\mathbf{x}_{b}$, the size of which is as small as $n_{\mathcal{B}}$ DOFs plus the number of linear normal modes for which the frequencies lie in the frequency ranges of interest. The use of such linear reduced-order modeling methods greatly helps analyze the dynamic response of systems with localized nonlinearities, such as transient dynamic analysis [35], and nonlinear harmonic response analysis [36]. However, even with these reducedorder modeling methods, if the number of DOFs involved in the $b$ partition becomes large (especially the cases with very fine mesh in the contacting regions), one cannot take advantage of the linear reduced-order modeling techniques, as the computational cost associated with the nonlinear dynamic analysis typically grows as the number of DOFs in the $b$ partition increases. Furthermore, if one simply attempts to eliminate some of the DOFs in the $b$ partition, it results in inaccurate, or even wrong, results. Therefore, to obtain accurate computational results, one needs to keep as many boundary DOFs as possible. That can easily result in prohibitively costly calculations. Typically, as a workaround to avoid the inaccurate results due to the lack of sufficient DOFs considered and, at the same time, to obtain an efficient computational model, one has to select the DOFs in a heuristic way, which greatly depends on the system characteristics and the analyst's experience and intuition. Moreover, if the model is developed in such ways, the error in the analysis results cannot be estimated a priori. Our aim here is to develop an automatic way to select the DOFs in $\mathcal{B}$ for a desired number of DOFs to be selected.

Note that the major assumptions made in the proposed methodology are typical for such approaches and can be summarized as follows:

1) The elastic structure is fixed in space, and the strain due to its vibration is infinitesimally small. Also, the nonlinearity comes purely from the intermittent contact at the contact surfaces, and it is localized. Other nonlinearities, such as large deformations or material nonlinearities, are not considered.
2) Contact surfaces $\Gamma_{A}$ and $\Gamma_{B}$ are invariant in time, and they are meshed as needed to ensure enough accuracy. Hence, the computational mesh is very fine, and the computational cost for solving the vibration problem involving the intermittent contact is prohibitively expensive. Therefore, the computational nodes have to be sampled, such that the resulting model has enough accuracy, yet the computational cost of using this model is as low as possible.

\section{A. Primary Model Reduction}

To reduce the number of DOFs included in $\mathcal{I}$ and make the subsequent development more efficient, a model reduction is first applied to Eq. (2). Namely, $\mathcal{I}$ is divided into two sets; that is, $\mathcal{I}=\mathcal{O} \cup \mathcal{D}$, where $\mathcal{O}$ is a set of DOF indices associated with the nodes to be directly used in the structural analysis, and $\mathcal{D}$ is the rest of DOF indices in $\mathcal{I}$, which is to be apparently deleted from the system by the reduction methods. In addition, a set of DOF indices to be used as the master DOFs is defined as active DOFs, designated as $\mathcal{A}$, and $\mathcal{A}=\mathcal{B} \cup \mathcal{O}$.

Next, consider an eigenvalue problem associated with Eq. (2), where the eigenvalue $\lambda$ and the corresponding eigenvector $\phi$ must satisfy

$$
\left(\begin{array}{ll}
\mathbf{K}_{a a} & \mathbf{K}_{a d} \\
\mathbf{K}_{d a} & \mathbf{K}_{d d}
\end{array}\right)\left[\begin{array}{l}
\boldsymbol{\phi}_{a} \\
\boldsymbol{\phi}_{d}
\end{array}\right]=\lambda\left(\begin{array}{ll}
\mathbf{M}_{a a} & \mathbf{M}_{a d} \\
\mathbf{M}_{d a} & \mathbf{M}_{d d}
\end{array}\right)\left[\begin{array}{l}
\boldsymbol{\phi}_{a} \\
\boldsymbol{\phi}_{d}
\end{array}\right]
$$

where $\boldsymbol{\phi}=\left[\boldsymbol{\phi}_{a}^{T}, \boldsymbol{\phi}_{d}^{T}\right]^{T}$. In this study, a mixed-boundary CMS of Hintz-Herting $[37, \underline{38}]$ is chosen for the primary model reduction. Namely, without the presence of rigid body modes, the coordinate transformation is defined as

$$
\left[\begin{array}{l}
\mathbf{x}_{a} \\
\mathbf{x}_{d}
\end{array}\right]=\mathbf{H} \boldsymbol{\eta}=\left(\begin{array}{ll}
\boldsymbol{\Psi} & \hat{\boldsymbol{\Phi}}
\end{array}\right)\left[\begin{array}{l}
\boldsymbol{\eta}_{a} \\
\boldsymbol{\eta}_{m}
\end{array}\right]
$$

where $\mathbf{x}_{a}=\boldsymbol{\eta}_{a}, \boldsymbol{\eta}_{m}$ is a vector of modal coordinates, $\boldsymbol{\Psi}$ and $\hat{\boldsymbol{\Phi}}$ are socalled constraint modes and truncated free-interface normal modes in a modified form that are, respectively, defined as

$$
\begin{gathered}
\boldsymbol{\Psi}=\left(\begin{array}{c}
\mathbf{I} \\
-\mathbf{K}_{d d}^{-1} \mathbf{K}_{d a}
\end{array}\right) \\
\hat{\boldsymbol{\Phi}}=\left(\begin{array}{c}
\mathbf{0} \\
\boldsymbol{\Phi}_{d}+\mathbf{K}_{d d}^{-1} \mathbf{K}_{d a} \boldsymbol{\Phi}_{a}
\end{array}\right)
\end{gathered}
$$

and $\boldsymbol{\Phi}=\left[\boldsymbol{\phi}_{(1)}, \boldsymbol{\phi}_{(2)}, \ldots, \boldsymbol{\phi}_{(k)}\right]$, where $k<n$; each subscript in parentheses denotes the corresponding mode number. Using the transformation defined in Eq. (4), the projected eigenvalue problem is obtained as

$$
\mathbf{K}_{H} \boldsymbol{\eta}=\mu \mathbf{M}_{H} \boldsymbol{\eta}
$$

where $\mathbf{M}_{H}=\mathbf{H}^{T} \mathbf{M H}$ and $\mathbf{K}_{H}=\mathbf{H}^{T} \mathbf{K H}$. It should be noted that the projected eigenvalue problem in Eq. (7) possesses at least the same eigenvalues of the original systems (i.e., $\lambda_{(1)}, \lambda_{(2)}, \ldots, \lambda_{\left(n_{m}\right)}$ ), although the indices may be different from the ones for the projected system. This is because the subspace spanned by the columns of $(\boldsymbol{\Psi}, \hat{\boldsymbol{\Phi}})$ contains the eigenvectors of Eq. (3) (i.e., $\boldsymbol{\phi}_{(j)} \in \operatorname{span}(\boldsymbol{\Psi}, \hat{\boldsymbol{\Phi}})$ for $j=1, \ldots, n_{m}$, as $\left.\operatorname{span}(\boldsymbol{\Psi}, \hat{\boldsymbol{\Phi}})=\operatorname{span}(\boldsymbol{\Psi}, \boldsymbol{\Phi})\right)$. Hence, the projected eigenvalue problem has the same eigenvalues as the original ones. Although this advantage comes with the expense of calculating the eigenvalues and eigenvectors of the full FE model, it is not a major drawback, considering that the computational cost involved in the nonlinear computations with the original FE would be much more expensive than calculating a few normal modes of the full FE model.

\section{B. Nonlinear Degree-of-Freedom Sampling}

With the reduced-order model obtained, as discussed in Sec. III.A, the next step is to select the DOFs in $\mathcal{B}$, such that the nonlinear characteristics of the system can be well approximated by applying the nonlinear BCs only at the selected DOFs. 
As mentioned, accurately calculating the NNM frequencies is the primary interest of this study. The NNM frequencies of the system can be obtained in several ways, such as by time integration of Eq. (2) for harmonic loading or by using harmonic-balance-based frequency/time domain analysis [36,39]. It was shown by the authors that the NNM frequencies for cracked plates, obtained by the nonlinear harmonic response analysis, can be well approximated by the application of bilinear frequency approximation, even when the crack surfaces involve multiple DOFs [40]. Therefore, as a measure to evaluate the results obtained with the selected DOFs, bilinear frequency is used in the following development. Namely, the $i$ th NNM frequency $\omega_{n i}$ can be approximated by a bilinear frequency $\omega_{b i}$ defined as

$$
\omega_{b i}=\frac{2 \omega_{o} \omega_{s}}{\omega_{o}+\omega_{s}}
$$

where $\omega_{o}$ and $\omega_{s}$ are the natural frequencies of the corresponding linear systems that can be, respectively, obtained by solving the following eigenvalue problems:

$$
\mathbf{K}_{H} \boldsymbol{\eta}=\left(\omega_{o}^{2}\right) \mathbf{M}_{H} \boldsymbol{\eta}
$$

subject to open BCs, and

$$
\mathbf{K}_{H} \boldsymbol{\eta}=\left(\omega_{s}^{2}\right) \mathbf{M}_{H} \boldsymbol{\eta}
$$

subject to sliding $\mathrm{BCs}$. The open $\mathrm{BC}$ is a boundary condition where no constraint is imposed on the nodes on $\Gamma_{A}$ and $\Gamma_{B}$, or the DOFs in $\mathcal{B}$. Thus, in fact, Eq. (9) is identical to Eq. (7). On the other hand, for the sliding $\mathrm{BC}$, it is assumed that $\Gamma_{A}$ can freely slide with respect to $\Gamma_{B}$ but cannot separate along the local normal direction, described as follows.

A contact pair is defined as a pair of nodes on $\Gamma_{A}$ and $\Gamma_{B}$ that may be in contact during the vibration. A set of numbers denoting all the contact pairs is defined as $\mathcal{C}_{c p}$. For the $j$ th contact pair in $\mathcal{C}_{c p}$, three mutually perpendicular normal vectors at a node on $\Gamma_{A}$ are defined as $\mathbf{n}_{1}^{j}, \mathbf{n}_{2}^{j}$, and $\mathbf{n}_{3}^{j}$, where $\mathbf{n}_{1}^{j}$ is the normal vector pointing outward from the surface, and $\mathbf{n}_{2}^{j}$ and $\mathbf{n}_{3}^{j}$ are unit vectors that are tangent to the surface and perpendicular to each other. Using these vectors, a coordinate transformation matrix $\mathbf{P}_{A}^{j}=\left(\mathbf{n}_{1}^{j}, \mathbf{n}_{2}^{j}, \mathbf{n}_{3}^{j}\right)$ is defined for each contact pair, with the assumption that a nodal displacement vector contains only translational DOFs, such that the $x_{1}$ component of the displacement vector of the node is aligned with $\mathbf{n}_{1}^{i}$ and points outward from the surface. For the other node of the $j$ th contact pair on $\Gamma_{B}$, the corresponding coordinate transformation matrix that aligns the $x_{1}$ component of the nodal displacement vector with the normal vector is defined as $\mathbf{P}_{B}^{j}=-\mathbf{P}_{A}^{j}$. Assembling $\mathbf{P}_{A}^{j}$ and $\mathbf{P}_{B}^{j}$ for all $j \in \mathcal{C}_{c p}$, a coordinate transformation is defined as

$$
\mathbf{P}=\left(\begin{array}{ccc}
\mathbf{P}_{b} & \mathbf{0} & \mathbf{0} \\
\mathbf{0} & \mathbf{I}_{o} & \mathbf{0} \\
\mathbf{0} & \mathbf{0} & \mathbf{I}_{m}
\end{array}\right), \quad \text { where } \mathbf{P}_{b}=A_{j=1}^{n_{\mathcal{C}_{c p}}}\left(\mathbf{P}_{A}^{j}, \mathbf{P}_{B}^{j}\right)
$$

and $A$ is an assembly operator, $\mathbf{P}_{b} \in \mathbb{R}^{n_{\mathcal{B}} \times n_{\mathcal{B}}}, \mathbf{I}_{o} \in \mathbb{R}^{n_{\mathcal{O}} \times n_{\mathcal{O}}}$, and $\mathbf{I}_{m} \in \mathbb{R}^{n_{\mathcal{M}} \times n_{\mathcal{M}}}$. Next, for the $j$ th contact pair, the $x_{1}$ components of the nodal displacement vectors, which are denoted as $\eta_{A}^{j}$ and $\eta_{B}^{j}$, are transformed to a relative displacement $u^{j} \triangleq\left(\eta_{A}^{j}+\eta_{B}^{j}\right) / \sqrt{2}$ and a displacement $v^{j} \triangleq\left(\eta_{A}^{j}-\eta_{B}^{j}\right) / \sqrt{2}$. Namely, denoting the set of DOFs corresponding to $u_{A}^{j}$ and $u_{B}^{j}$ for all $j$ th contact pair, one obtains

$$
\left[\begin{array}{l}
u^{j} \\
v^{j}
\end{array}\right]=\frac{1}{\sqrt{2}}\left(\begin{array}{cc}
1 & 1 \\
1 & -1
\end{array}\right)\left[\begin{array}{l}
\eta_{A}^{j} \\
\eta_{B}^{j}
\end{array}\right]
$$

Now, by defining $\mathcal{X}, \mathcal{Y}$, and $\mathcal{Z} \quad(\mathcal{X} \cup \mathcal{Y} \cup \mathcal{Z}=\mathcal{B}$ and $n_{\mathcal{X}}=n_{\mathcal{Y}}=n_{\mathcal{Z}}=n_{\mathcal{C}_{c p}}$ ), the sets that, respectively, contain sets of indices of the DOFs corresponding to $x_{1}, x_{2}$, and $x_{3}$ for all $j \in \mathcal{C}_{c p}$, and denoting the coefficient matrix in the Eq. (12) as $\mathbf{R}^{j}$, one can define a transformation matrix $\mathbf{R}$ by assembling $\mathbf{R}^{j}$ for $j \in \mathcal{C}_{c p}$ as follows:

$$
\mathbf{R}=\left(\begin{array}{cc}
\mathbf{R}_{x} & \mathbf{0} \\
\mathbf{0} & \mathbf{I}
\end{array}\right), \quad \text { where } \mathbf{R}_{x}=A_{j=1}^{n_{\mathcal{C}_{c p}}}\left(\mathbf{R}^{j}\right)
$$

and $\mathbf{R}_{x} \in \mathbb{R}^{n_{\mathcal{X}} \times n_{\mathcal{X}}}$. Since $\mathbf{P}^{-1}=\mathbf{P}^{T}$ and $\mathbf{R}^{-1}=\mathbf{R}^{T}$, the eigenvalue problem in Eq. (7) can be transformed to

$$
\mathbb{K} \mathbf{q}=\left(\omega_{o}^{2}\right) \mathbb{M} \mathbf{q}
$$

where $\boldsymbol{\eta}=\mathbf{P R q}, \mathbb{M}=(\mathbf{P R})^{T} \mathbf{M}_{H} \mathbf{P R}$, and $\mathbb{K}=(\mathbf{P R})^{T} \mathbf{K}_{H} \mathbf{P R}$. Next, note that $\mathbf{q}$ can be partitioned into $\mathbf{q}=\left[\mathbf{q}_{r}, \mathbf{q}_{g}\right]$, where $\mathbf{q}_{r}$ is the vector of relative DOFs, or $u^{j}\left(\forall j \in \mathcal{C}_{c p}\right)$ and $\mathbf{q}_{g}$ is the generalized internal DOFs containing $v^{j}\left(\forall j \in \mathcal{C}_{c p}\right)$, the $x_{2}$, and $x_{3}$ components of the nodal displacement vectors of the nodes of the contact pairs, the displacement vectors of the observer nodes, and the modal coordinates. That is, Eq. (14) can be written as

$$
\left(\begin{array}{ll}
\mathbb{K}_{r r} & \mathbb{K}_{r g} \\
\mathbb{K}_{g r} & \mathbb{K}_{g g}
\end{array}\right)\left[\begin{array}{l}
\mathbf{q}_{r} \\
\mathbf{q}_{g}
\end{array}\right]=\left(\omega_{o}^{2}\right)\left(\begin{array}{ll}
\mathbb{M}_{r r} & \mathbb{M}_{r g} \\
\mathbb{M}_{g r} & \mathbb{M}_{g g}
\end{array}\right)\left[\begin{array}{l}
\mathbf{q}_{r} \\
\mathbf{q}_{g}
\end{array}\right]
$$

where $\mathcal{R} \subset \mathcal{X}$ and $\mathcal{G}=(\mathcal{A} \backslash \mathcal{R}) \cup \mathcal{M}$.

The best approximation to the NNM frequency can be obtained when the sliding BCs are imposed on all of the nodes on the surfaces $\Gamma_{A}$ and $\Gamma_{B}$. Namely, the associated eigenvalue problem with the sliding BCs can be obtained by constraining all the relative DOFs, or $\mathbf{q}_{r}=\mathbf{0}$; that is,

$$
\mathbb{K}_{g g} \mathbf{q}_{g}=\left(\omega_{s}^{2}\right) \mathbb{M}_{g g} \mathbf{q}_{g}
$$

Next, we assume that we do not like to consider all nodes in $\mathcal{R}$ for the subsequent forced response analysis due to the large number of DOFs involved in $\mathcal{R}$. In other words, the nodes where the nonlinear BCs are applied should be sampled, as illustrated in Fig. 2. The selected DOFs are designated as nonlinear DOFs, and a set of indices of the nonlinear DOFs is denoted by $\mathcal{N}$, where $\mathcal{N} \subset \mathcal{R}$. The rest of the DOFs in $\mathcal{R}$ are designated as linear DOFs, and the associated set is denoted by $\mathcal{L}$, where $\mathcal{N} \cup \mathcal{L}=\mathcal{R}$. Therefore, the bilinear frequency is calculated with $\omega_{s}$, such that the sliding $\mathrm{BC}$ is applied only on the DOFs in $\mathcal{N}$, or $\mathbf{q}_{n}=\mathbf{0}$; that is,

$$
\left(\begin{array}{ll}
\mathbb{K}_{l l} & \mathbb{K}_{l g} \\
\mathbb{K}_{g l} & \mathbb{K}_{g g}
\end{array}\right)\left[\begin{array}{l}
\mathbf{q}_{l} \\
\mathbf{q}_{g}
\end{array}\right]=\left(\omega_{s}^{2}\right)\left(\begin{array}{cc}
\mathbb{M}_{l l} & \mathbb{M}_{l g} \\
\mathbb{M}_{g l} & \mathbb{M}_{g g}
\end{array}\right)\left[\begin{array}{l}
\mathbf{q}_{l} \\
\mathbf{q}_{g}
\end{array}\right]
$$

Considering that the natural frequencies of the system with the open BCs, $\omega_{o}$, are independent of both the number and the pattern of the selected DOFs [recalling that $\operatorname{span}\left(\boldsymbol{\Psi}_{c}, \hat{\Phi}\right)$ contains the chosen eigenvectors], one can see from Eq. ( $\underline{8})$ that $\omega_{b i}$ is dependent only on $\omega_{s}$ for a fixed $\omega_{o}$.

Next, considering the Rayleigh's theorem of constraints, defined by Eq. (1), it is known that the system's eigenvalues increase if a constraint is imposed on a system. Therefore, as the number of constraints on Eq. (7) increases, $\omega_{s}$ increases. Furthermore, $\omega_{b i}$ is a monotonically increasing function of $\omega_{s}$ for a fixed $\omega_{o}$, because $\partial \omega_{b i} / \partial \omega_{s}=2 \omega_{o}^{2} /\left(\omega_{o}+\omega_{s}\right)^{2} \geqslant 0$. Hence, one can conclude that the best approximation of $\omega_{b i}$ for a given $n_{\mathcal{N}}$ is obtained when the maximum $\omega_{s}$ is achieved. Thus, a corresponding maximization problem is stated as follows:

$$
\max _{\mathcal{N} \subset \mathcal{R}} \omega_{s}(\mathcal{N}), \quad \text { subject to }|\mathcal{N}|=n_{\mathcal{N}}
$$

This maximization problem may be solved by mathematical programming methods, such as integer programming or topology optimization methods, as was done in [27]. As shall be discussed next, this maximization problem can in fact be treated in a more

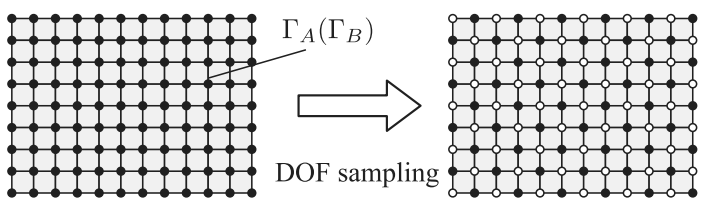

Fig. 2 Schematic of the node sampling: $\bullet$, selected node $(\mathcal{N})$. 
efficient way by the use of Guyan reduction [5] and methods to choose the master DOFs for reduced-order modeling techniques.

\section{Automatic Master Degree-of-Freedom Selection}

The methods for automatically selecting the master DOFs for Guyan reduction [5] have been previously developed $[9,10,16]$. In particular, the method proposed by Henshell and Ong [9] appears to be the most successful approach. Although it has been known to be computationally expensive, due to the nature of eliminating a single DOF per iteration and the need for successive applications of Guyan reduction [5], this can be alleviated by the use of the primary model reduction by CMS, as developed in Sec. III.A. As was mentioned by Bouhaddi and Fillod [16] and Shah and Raymund [10], the master DOFs of Guyan reduction [5] should be chosen such that the valid eigenvalue range of the reduced-order model is maximized. In general, it has been known that the eigenvalue range of validity is bounded by the lowest eigenvalue of the system, with all the master DOFs fixed. Here, this concept is applied to the problem of finding the optimal $\mathcal{N}$ that solves Eq. (18). Namely, the corresponding eigenvalue problem is Eq. (17), where $\mathbf{q}_{n}$ is regarded as the master DOFs. As was discussed in [41], the error bounds in the $i$ th eigenvalue of the reduced model produced by the Guyan reduction [5] can be obtained a priori by the following relationship:

$$
0 \leqslant \varepsilon_{i} \leqslant \frac{\lambda_{i}}{\lambda_{s, \min }-\lambda_{i}}
$$

where $\varepsilon_{i} \triangleq\left(\bar{\lambda}_{i}-\lambda_{i}\right) / \lambda_{i}$ is the relative error in the $i$ th eigenvalue, $\bar{\lambda}_{i}$ is the $i$ th eigenvalue of the reduced-order model, $\lambda_{i}$ is the $i$ th eigenvalue of the original FE model, and $\lambda_{s, \min }$ is the smallest eigenvalue of the system with all the master DOFs fixed. For $\lambda_{i} / \lambda_{s, \min } \ll 1$, the upper bound asymptotically converges to the following value [르]:

$$
0 \leqslant \varepsilon_{i} \leqslant \lambda_{i} / \lambda_{s, \min }
$$

Therefore, it is apparent that maximizing $\lambda_{s, \min }$ results in minimizing the upper bound of the error for all the eigenvalues of the reducedorder model. Hence, this provides a guideline for selecting the master DOFs for Guyan reduction [5], such that the errors in the eigenvalues of the resulting reduced-order model are minimized.

By observing this fact from another point of view, one may note that, if a certain set of master DOFs can achieve the maximum $\lambda_{s, \min }$, one can obtain not only an accurate reduced-order model that can well approximate the first few lowest eigenvalues of the original system but also (as a by-product) a good estimate on the optimal constraint locations that maximize the fundamental frequency. Recasting this to the original problem of selecting the optimal set $\mathcal{N}$, the error bounds given in Eq. (20) and associated with the eigenvalue problem in Eq. (17) are written as

$$
0 \leqslant \varepsilon_{i} \leqslant \frac{\left(\omega_{o}^{2}\right)_{i}}{\left(\omega_{s}^{2}\right)_{1}-\left(\omega_{o}^{2}\right)_{i}}
$$

where $\varepsilon_{i} \triangleq\left[\left(\bar{\omega}_{o}^{2}\right)_{i}-\left(\omega_{o}^{2}\right)_{i}\right] /\left(\omega_{o}^{2}\right)_{i},\left(\bar{\omega}_{o}^{2}\right)_{i}$ is the $i$ th eigenvalue of a reduced-order model, and $\left(\omega_{s}\right)_{1}$ is the lowest natural frequency of Eq. (17). The corresponding maximization problem is given by Eq. ( $(\overline{18})$. Solving this problem for the lowest eigenvalue $\left(\omega_{s}\right)_{1}$, one can expect that the chosen nodes pattern is at least quasi-optimal.

According to $[42,43]$, the sequential elimination method by Henshell and Ong [9] tends to keep $\lambda_{s, \min }$ high, as it eliminates the DOFs associated with the highest constrained frequency at each iteration as the slave DOFs. Namely, after the elimination procedure, if the chosen master DOFs are all fixed, the system is left with the (slave) DOFs that were identified to have the highest constrained frequency at each elimination process. Thus, the resulting system with all the master DOFs fixed tends to have a larger $\lambda_{s, \min }$ than that calculated with systems with other possible combinations of master DOFs fixed.

The Henshell and Ong [9] method that is adapted specifically for this problem is shown in Algorithm $\underline{1}$. First, at each iteration, the ratios of the diagonal terms of the stiffness matrix $k_{j j}$ to the diagonal terms of the mass matrix $m_{j j}$ are calculated for $\forall j \in \mathcal{R}$. Next, the index $q_{1}$ that gives the maximum ratio among $j \in \mathcal{R}$ is obtained. Next, the set $\mathcal{L}$ is updated, such that it contains $q_{1}$ and all the other DOFs that are associated with the contact pair $k \in \mathcal{C}_{c p}$ to which the $q_{1}$ th DOFs belongs (e.g., the DOFs that are perpendicular to the normal direction). The set $\mathcal{N}$ is then updated, such that it excludes the selected DOFs of $\mathcal{L}$ from $\mathcal{R}$, and the set $\mathcal{R}$ is redefined as $\mathcal{N}$. A constraint mode is calculated by solving a problem where a unit displacement is applied to a DOF in $\mathcal{N}$, whereas all the other DOFs in $\mathcal{N}$ are fixed. This is repeated for all DOFs in $\mathcal{N}$, resulting in the following matrix:

$$
\boldsymbol{\Psi}=\left(\begin{array}{c}
\mathbf{I} \\
-\left(\mathbb{K}_{l l}\right)^{-1} \mathbb{K}_{l n}
\end{array}\right)
$$

where $\boldsymbol{\Psi}$ is the matrix of constraint modes for all DOFs in $\mathcal{N}$. Guyan reduction [5] is then applied to the mass and stiffness matrices. The iteration continues until the number of DOFs in $\mathcal{N}$ reaches the desired value for $n_{\mathcal{N}}$.

To demonstrate the performance of the proposed algorithm, another algorithm for selecting DOFs is shown here for comparison. The method of effective independence vector, or the EIDV method developed by Kammer [26], is a method to choose the sensor placement locations for the vibration measurement of large-scale structures. The method aims to make the measured, or sampled/ truncated eigenvectors, as linearly independent as possible. According to Penny et al. [25], many of the criteria for choosing the master DOFs for model-order reduction are similar to choosing measurement locations in a way such that the lower-frequency modes can be captured accurately. In fact, as examined by Penny et al., both the Henshell and Ong [9] method and the EIDV method produce acceptable selections in most cases (in a quasi-optimal manner). The DOFs selection algorithm based on the EIDV method is shown as Algorithm 2. First, the eigenvalue problem in Eq. (16) is solved for the first $k$ modes, and the associated modal matrix is denoted by $\boldsymbol{\Phi}_{k}=\left(\boldsymbol{\phi}_{1}, \boldsymbol{\phi}_{2}, \ldots, \boldsymbol{\phi}_{k}\right)$. That is, $\mathbb{K} \boldsymbol{\Phi}_{k}=\mathbb{M} \boldsymbol{\Phi}_{k} \boldsymbol{\Lambda}_{k}$, where $\boldsymbol{\Lambda}_{k}$ is a

\begin{tabular}{|c|c|}
\hline & m 1 DO \\
\hline 1: & $\begin{array}{l}\text { for } i=1 \text { to } i=n_{\mathcal{R}}-n_{\mathcal{N}}, \text { do } \\
\text { Calculate } \sqrt{k_{j j} / m_{j j}} \text { for } j \in \mathcal{R}\end{array}$ \\
\hline 3: & Find $q_{1}$, such that $\sqrt{k_{q_{1} q_{1}} / m_{q_{1} q_{1}}}=\max _{j \in \mathcal{R}} \sqrt{k_{j j} / m_{j j}}$ \\
\hline 4: & $\begin{array}{c}\mathcal{L} \leftarrow\left\{q_{1}, \ldots, q_{n_{k}}\right\}, \text { where } q \mathrm{~s} \text { are the DOFs associated with the } k \text { th } \\
\text { contact pair }\left(k \in \mathcal{C}_{c p}\right) \text { and } n_{k} \text { is the number of DOFs } \\
\text { in the } k \text { th contact pair }\end{array}$ \\
\hline 5: & $\mathcal{N} \leftarrow \mathcal{R} \backslash \mathcal{L}$ \\
\hline 6: & $\mathcal{R} \leftarrow \mathcal{N}$ \\
\hline 7 & nstraint modes \\
\hline 8: & $\begin{array}{l}\text { Apply Guyan reduction [5] to the system matrices: } \mathbb{M} \leftarrow \Psi^{T} \mathbb{M} \\
\text { and } \mathbb{K} \leftarrow \Psi^{T} \mathbb{K} \Psi\end{array}$ \\
\hline 9: & end for \\
\hline
\end{tabular}
diagonal matrix, with diagonal entries being the square of the natural frequencies of the open BC linear system. The Fisher information matrix $\mathbf{A}$ is then calculated as $\mathbf{A}=\boldsymbol{\Phi}_{k}^{T} \boldsymbol{\Phi}_{k}$, and an idempotent matrix

Algorithm 2 DOF selection based on EIDV method

\begin{tabular}{lc}
\hline \hline 1: & Calculate $\boldsymbol{\Phi}_{k}$ \\
$2:$ & for $i=1$ to $i=n_{\mathcal{R}}-n_{\mathcal{N}}$, do \\
$3:$ & $\mathbf{A} \leftarrow \boldsymbol{\Phi}_{k}^{T} \boldsymbol{\Phi}_{k}$ \\
4: & $\mathbf{E} \leftarrow \boldsymbol{\Phi}_{k} \mathbf{A}^{-1} \boldsymbol{\Phi}_{k}^{T}$ \\
5: & Find $q_{1}$, such that $e_{q_{1} q_{1}}=\min _{j \in \mathcal{R}} e_{j j}$ \\
6: & $\mathcal{L} \leftarrow\left\{q_{1}, \ldots, q_{n_{k}}\right\}$, where $q$ s are the DOFs associated with the $k$ th \\
& contact pair $\left(k \in \mathcal{C}_{c p}\right)$ and $n_{k}$ is the number of DOFs in $k$ th \\
& contact pair \\
7: & $\mathcal{N} \leftarrow \mathcal{R} \backslash \mathcal{L}$ \\
8: & $\mathcal{R} \leftarrow \mathcal{N}$ \\
$9:$ & Delete rows of $\boldsymbol{\Phi}_{k}$ corresponding to the DOFs in $\mathcal{L}$ \\
10: & end for \\
\hline \hline
\end{tabular}




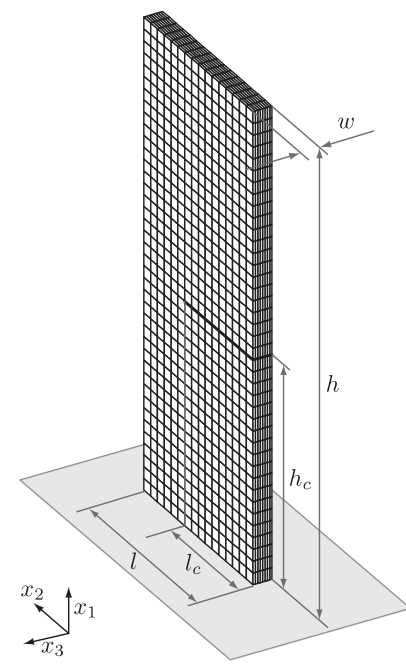

a) FE model

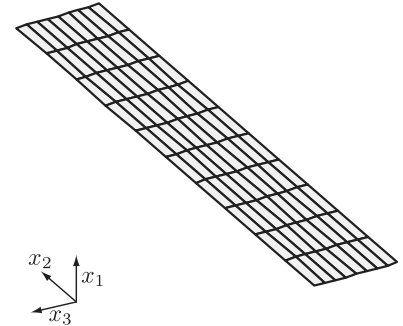

b) Magnified crack surface
Fig. 3 Cantilevered cracked plate model.

$\mathbf{E}$ is computed as $\mathbf{E}=\boldsymbol{\Phi}_{k} \mathbf{A}^{-1} \boldsymbol{\Phi}_{k}^{T}$. The diagonal of $\mathbf{E}$ is called the independence distribution vector (see [26] for detailed formulations). The least contributing DOF to the independence of the modes among the ones in $\mathcal{R}$ is identified as the one with the smallest diagonal element in $\mathbf{E}$. The associated DOFs are also identified and stored in $\mathcal{L}$, and both $\mathcal{N}$ and $\mathcal{R}$ are updated, as in the Henshell and Ong [9] method. Finally, the rows of $\boldsymbol{\Psi}_{k}$ corresponding to the DOFs in $\mathcal{L}$ are deleted. The iteration continues until the size of $\mathcal{N}$ reaches the desired $n_{\mathcal{N}}$.

Although the EIDV method has a similar objective for choosing DOFs as the Henshell and Ong [9] method, the objective of the EIDV method is not exactly the maximization problem of Eq. (18). Therefore, it is expected that the Henshell and Ong method returns better solutions to the given maximization problem than the EIDV method, as is shown in the next section.

\section{Case Studies}

In Sec. III, the method to select the nonlinear DOFs has been introduced. In this section, the performance and accuracy of the method are demonstrated by applying the algorithm to two example problems. In the first case study, the accuracy of the proposed method is discussed in terms of the bilinear frequencies and forced response. Furthermore, a metric to assess accuracy is introduced and examined. The second case study is provided to demonstrate the performance of the proposed method when applied to a system with a more realistic and complex FE model featuring a large number of DOFs on the faces involving intermittent contact.

\section{A. Simple Cracked Plate Model}

1. Problem Description

A cantilevered cracked plate model was constructed with Young's modulus of $E=2.0 \times 10^{11} \mathrm{~Pa}$, Poisson's ratio of $v=0.3$, density of $\rho=7800 \mathrm{~kg} / \mathrm{m}^{3}$, and geometry, shown in Fig. 3a, where $w=6.0 \times$ $10^{-3} \mathrm{~m}, l=6.0 \times 10^{-2} \mathrm{~m}, h=1.5 \times 10^{-1} \mathrm{~m}, l_{c} / l=0.625$, and $h_{c} / h=0.475$. The model was discretized with 5120 linear solid elements and resulted in mass and stiffness matrices with 18,630 DOFs. On the crack surfaces, shown in Fig. 3b, there are 180 nodes, or 90 contact pairs, on the surfaces. Hence, the number of associated DOFs is 540. The CMS method, shown in the Sec. III.A was then applied to the FE model, and it resulted in a 681 DOF system $(3.6 \%$ of the original size) consisting of 621 physical DOFs and 60 modal coordinates, corresponding to the free-interface normal modes. With this reduced-order model, both algorithms in Algorithms 1 and 2 were applied for $n_{\mathcal{N}}=4,8,16,32,64$, and 128. For the EIDV algorithm, the first four modes were considered to construct the modal matrix.

To compare these results with an intuitive selection method, a selection criterion was also employed, where the nonlinear DOFs were chosen based on the amount of penetration between the nodes in a contact pair for the modes of interest (the fourth mode), which is referred to as the penetrating surface criterion. Namely, the penetrating surface criterion is based on the observation that penalizing the interpenetration of the most penetrating contact pairs may

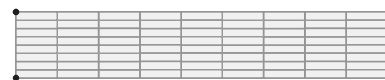

a) $n_{N}=4$ (2 pairs)

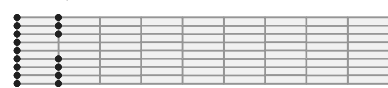

d) $n_{N}=32$ (16 pairs)



b) $n_{N}=8$ (4 pairs)

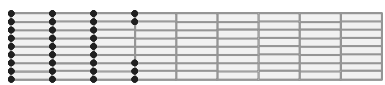

e) $n_{N}=64$ (32 pairs)

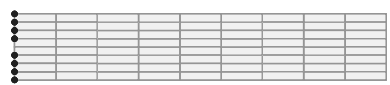

c) $n_{N}=16(8$ pairs $)$

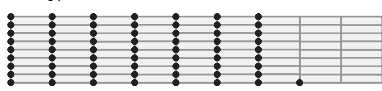

f) $n_{N}=128$ (64 pairs)

Fig. 4 Selected nodes by penetrating surface criterion (left edge open).
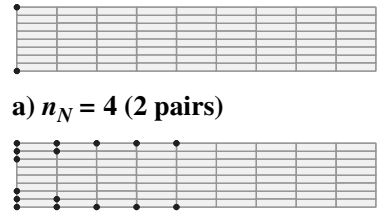

d) $n_{N}=32$ (16 pairs)

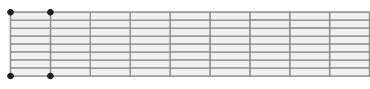

b) $n_{N}=8$ (4 pairs)

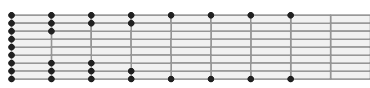

e) $n_{N}=64$ (32 pairs)

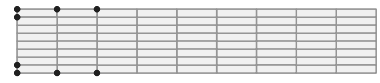

c) $n_{N}=16(8$ pairs $)$

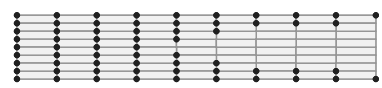

f) $n_{N}=128$ (64 pairs)

Fig. 5 Selected nodes by EIDV method (left edge open).

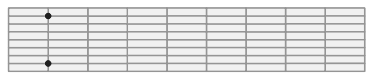

a) $n_{N}=4$ (2 pairs)

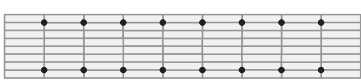

d) $n_{N}=32$ (16 pairs)

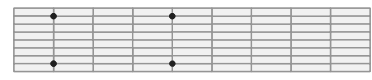

b) $n_{N}=8$ (4 pairs)

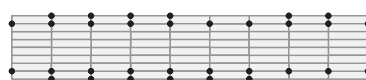

e) $n_{N}=64$ (32 pairs)

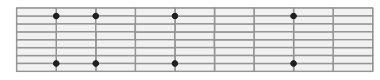

c) $n_{N}=16(8$ pairs $)$

: : : : : : : : : :

: : : : : : : : : : : :

f) $n_{N}=128$ (64 pairs)

Fig. 6 Selected nodes by the Henshell and Ong [9] method (left edge open). 
produce the stiffest system response. The selected node pattern with such criterion is shown in Fig. 4, and the results of the EIDV method and the Henshell and Ong [9] method are shown in Figs. $\underline{5}$ and $\underline{6}$. As can be seen in Fig. 4, if the nodes are chosen based on the penetrating surface criterion, the selection starts from the nodes near the crack edge (open side) for $n_{\mathcal{N}}=4$, and it then proceeds toward the tip of the crack (closed side) as $n_{\mathcal{N}}$ increases. This result is expected, because the motion of the crack surface is more significant near the open edge than near the closed edge. In contrast, the EIDV method starts to select nodes near the crack edge, but it tends to choose more nodes on the crack rims than nodes near the crack edge, as shown in Fig. 5. Finally, the Henshell and Ong method selects the nodes near the crack edge first, for $n_{\mathcal{N}}=4$, but it then tends to select nodes over the crack surface in a more distributed manner, as can be seen in Fig. $\underline{6}$.

\section{Forced Response Analysis}

Next, to evaluate the influence of the application of the nonlinear BC onto the selected nodes on an NNM frequency, forced response analysis was carried out by applying an external harmonic loading to the cracked plate. As one may note, the repetitive opening and closing of the crack faces must be treated appropriately with contact algorithms when the forced response of this cracked structure is considered. As a result, the vibration is nonlinear and the steady-state response may not be expressed as a harmonic function, even if the external force is a harmonic function. Therefore, in this study, the steady-state response was obtained by assuming that the displacement can be expressed as a truncated Fourier series, and the nonlinear BC can be enforced by a penalty method [1]. The solution method is also known as the hybrid frequency time domain method [36,39], which is based on the concept of the harmonic balance method [4]. The detailed formulation of the method is omitted in this paper for the sake of brevity.

It is noted that the system matrices were further reduced by the application of Eq. (4) to the reduced-order model before the forced response calculation was performed. That was done by keeping the selected node pairs as active DOFs and condensing out the other DOFs, including physical and modal coordinates. For example, with
$n_{\mathcal{N}}=64$ (32 pairs), the system size was reduced down to 155 DOFs, which is $0.83 \%$ of the original system size.

A harmonic forcing of magnitude $3 \mathrm{~N}$ was then applied at the tip of the plate to excite the first vibration mode, which is the first out-ofplane bending mode. The forced response was calculated for both the linear case (i.e., with open BC) and the nonlinear case (with the nonlinear BCs imposed on $\mathcal{N}$ ). The results are shown in Fig. 7. As can be seen in Fig. 7a, the selection pattern based on the Henshell and Ong [9] method does not alter the linear forced response. This is because the selection of the active DOFs does not alter the eigenvalues of the linear reduced-order model. In contrast, the number of contact pairs greatly affects the results of nonlinear forced response, as shown in Fig. 7b. Also, one may observe that the response obtained with 64 contact pairs is almost identical to that obtained with the full set of 90 contact pairs. That implies that, for accurately calculating the nonlinear resonant frequencies, it may not be necessary to enforce nonlinear BCs for all the contact pairs on the crack faces. The same forced response calculations were carried out with the node patterns selected by the EIDV method and the penetrating surface criterion. The results obtained are shown in Figs. 7c and 7d. As can be seen in Fig. 7c, the results obtained with the patterns chosen by the EIDV method are comparable with the ones produced by the Henshell and Ong method. Also, as can be seen in Fig. 7d, the forced response with the node patterns chosen by the penetrating surface criterion produced worse results than the other two methods (i.e., for a given number of $n_{\mathcal{N}}$, the predicted resonant frequency by the approach is less accurate than that calculated by the other methods). This is the most visible in the results for $n_{\mathcal{N}}=64$, for which both the Henshell and Ong method and the EIDV method produced results that are almost identical to the results for the full model with $n_{\mathcal{N}}=90$.

Moreover, to evaluate the effects of $n_{\mathcal{N}}$ on the computational speed, the average CPU times for generating node patterns, as well as the ones required to obtain the steady-state forced response, are shown in Table 1 . The CPU time was measured on a computer with an Intel Core 2 Dū $2.4 \mathrm{GHz}$ processor and $4.0 \mathrm{~GB}$ of RAM. First, the CPU time for generating the node pattern with each algorithm was measured for 100 trials, and the average values are shown in Table 1 .

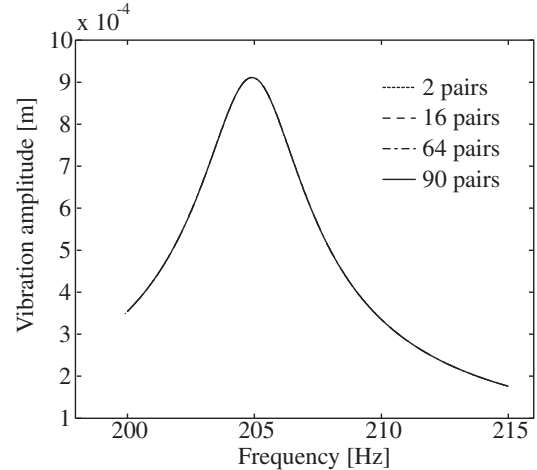

a)Henshell and Ong method [9] (linear)

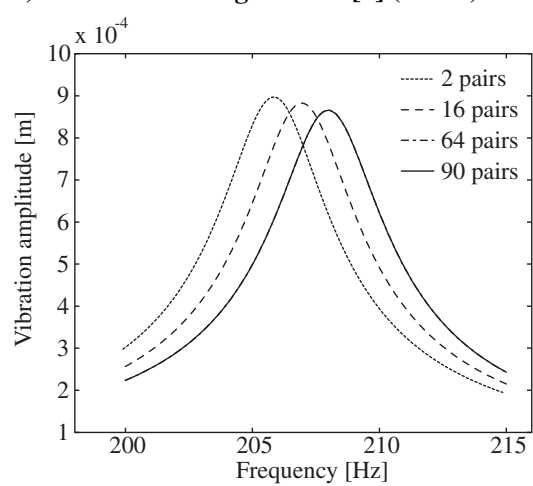

c) EIDV method (nonlinear)

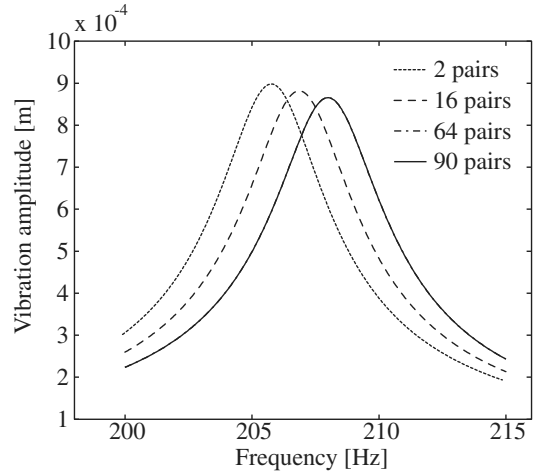

b) Henshell and Ong method [9] (nonlinear)

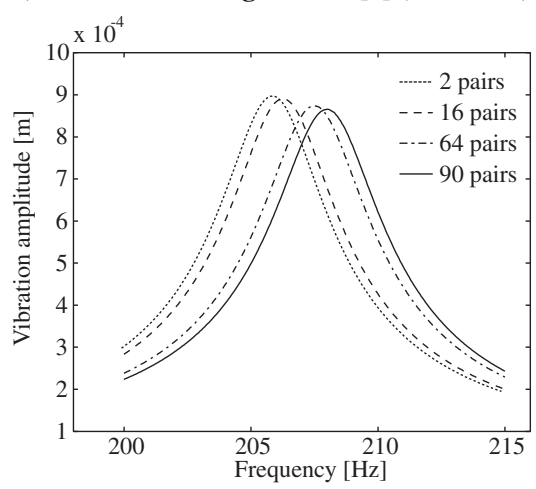

d) Penetrating surface criterion (nonlinear)

Fig. 7 Results of forced response analysis of the cracked plate. 
Table 1 Average CPU times for generating node patterns over 100 trials, and typical CPU times to compute forced response for 256 steps within 200 to $215 \mathrm{~Hz}$

\begin{tabular}{ccccccc}
\hline & \multicolumn{2}{c}{ Penetrating surface criterion } & \multicolumn{2}{c}{ EIDV } & \multicolumn{2}{c}{ Henshell and Ong [9] } \\
\cline { 2 - 6 }$n_{\mathcal{N}}$ & Node selection, s & Forced response, s & Node selection, s & Forced response, s & Node selection, s & Forced response, s \\
\hline 2 & $6.15 \times 10^{-1}$ & 1.27 & 2.23 & 1.18 & 3.69 & 1.21 \\
4 & $6.21 \times 10^{-1}$ & 5.64 & 2.26 & 3.99 & 3.68 & 3.23 \\
8 & $6.18 \times 10^{-1}$ & $1.85 \times 10^{1}$ & 2.19 & $2.87 \times 10^{1}$ & 3.69 & $2.07 \times 10^{1}$ \\
16 & $6.07 \times 10^{-1}$ & $5.00 \times 10^{1}$ & 2.20 & $1.07 \times 10^{2}$ & 3.65 & $9.35 \times 10^{1}$ \\
32 & $6.09 \times 10^{-1}$ & $1.48 \times 10^{3}$ & 2.12 & $1.37 \times 10^{3}$ & 3.52 & $8.17 \times 10^{2}$ \\
64 & $6.26 \times 10^{-1}$ & $1.08 \times 10^{4}$ & 1.93 & $9.86 \times 10^{3}$ & 2.66 & $1.03 \times 10^{4}$ \\
90 & N/A & $2.62 \times 10^{4}$ & N/A & $2.62 \times 10^{4}$ & N/A & $2.62 \times 10^{4}$ \\
\hline \hline
\end{tabular}

As can be seen, the node selection by the Henshell and Ong [9] method takes longer than the other two methods for all $n_{\mathcal{N}}$, due mostly to the cost associated with the successive application of Guyan reduction [5]. The other two methods are faster than the Henshell and Ong [9] method. However, they sacrifice the accuracy in the resulting nonlinear forced response. Also note that the CPU time decreases as $n_{\mathcal{N}}$ increases for both EIDV and Henshell and Ong methods, because these methods eliminate the unnecessary DOFs at each iteration rather than choosing necessary DOFs. Thus, the node pattern with larger $n_{\mathcal{N}}$ is generated with less CPU time. Second, the forced response was calculated at 256 points evenly spaced in a frequency range from 200 to $215 \mathrm{~Hz}$ for $n_{\mathcal{N}}=2,4,8,16,32$, and 64 by the three methods considered, and for the case with $n_{\mathcal{N}}=90$, and the results are shown in Table 1. Although there is no significant CPU time difference among the selection algorithms, one can notice that the CPU time can be greatly reduced when the calculations are done with the sampled nodes, without sacrificing too much accuracy if the set of nodes were chosen by the proposed method. Also, note that the node selection has to be done only once for the entire frequency range of interest, and the cost is much smaller (e.g., only $0.03 \%$ for the Henshell and Ong method with $n_{\mathcal{N}}=64$ ) than that required for computing forced response with sufficiently large $n_{\mathcal{N}}$.

\section{Bilinear Frequency Approximation}

Next, the influence of the selected node pattern on the bilinear frequencies is discussed. The first four bilinear frequencies were calculated for the model with the selected node patterns with the three node sampling methods, and the results are shown in Fig. 8 along with their linear vibration mode shapes. The first four modes correspond to the first out-of-plane bending, the first torsion, the second out-of-plane bending, and the first in-plane bending modes, respectively. The plots in Fig. $\underline{8}$ show the percentage errors in the bilinear frequency versus the number of contact pairs, where the error is defined as the ratio of the difference between the bilinear frequency with the sampled contact pairs and the frequency obtained with the full set of contact pairs, to that with the full set of contact pairs. As can



a)

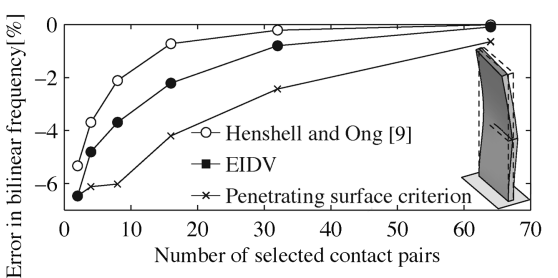

c) be seen in Fig. 8, the Henshell and Ong [9] method consistently provides the best results among all the methods for the first four modes. Moreover, it shows the best convergence rate in terms of the number of contact pairs.

\section{A Posteriori Accuracy Assessment}

Even though intuitive, the approach that chooses the contact pairs with the most penetration does not provide the best results, because applying the nonlinear BCs at these nodes does not result in the stiffest vibration response. In contrast, the Henshell and Ong [9] method and the EIDV method produced node patterns that yield results closer to the reference results in terms of forced response and bilinear frequencies. In particular, the Henshell and Ong method iteratively aims to solve the maximization problem in Eq. (18) in a quasi-optimal manner. Therefore, the bilinear frequencies, as well as the resonant frequencies, were well approximated with the nodes chosen by the Henshell and Ong method.

To better understand the governing factor for the accuracy of the results, a more physical interpretation of the results is provided here. Namely, the key effect for achieving the good approximation of the NNM frequency is to ensure, as much as possible, the nonpenetrability condition on the contact pairs where the nonlinear BCs are not applied. Also, the penetration should be evaluated during a complete vibration cycle. Thus, both the depth and the duration of the penetration should be taken into account. These quantities vary in space and depend on the frequency of vibration. Hence, as a metric to characterize not only the amount but also the duration of penetration over the entire crack surfaces for a given vibration frequency, the following quantity is introduced:

$$
\hat{F}=\int_{0}^{T}\left[\int_{\Gamma_{A}\left(\Gamma_{B}\right)} k_{e} u_{p}(\mathbf{r}, t) \mathrm{d} \Gamma\right] \mathrm{d} t
$$

where $\hat{F}$ is a quantity with the dimension of impulse referred to as the virtual impulse, $k_{e}$ is an equivalent spring constant per unit length determined by the ratio between the Young's modulus multiplied by

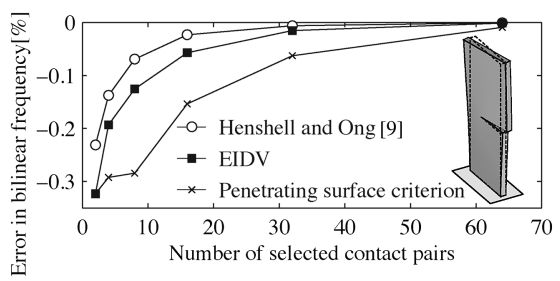

b)

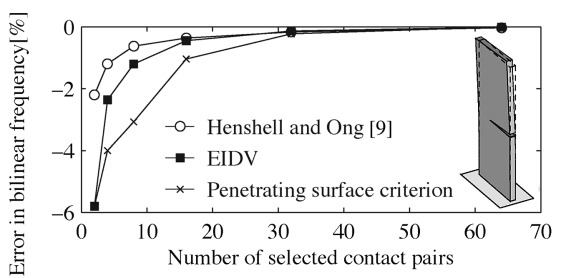

d)

Fig. 8 Errors in the first four bilinear frequencies: a) NNM 1, b) NNM 2, c) NNM 3, and d) NNM 4. 

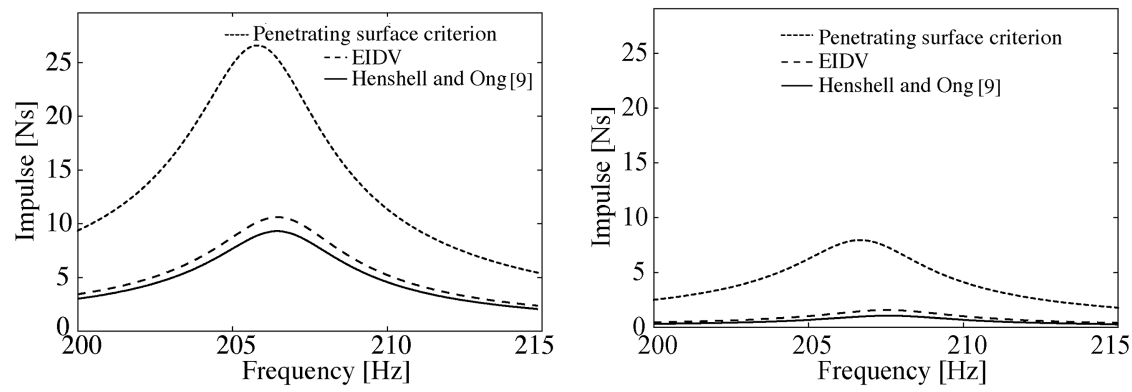

a) $n_{N}=16$ (8 pair)

b) $n_{N}=64$ ( 32 pair)

Fig. 9 Virtual impulse for a period of vibration for NNM 1.

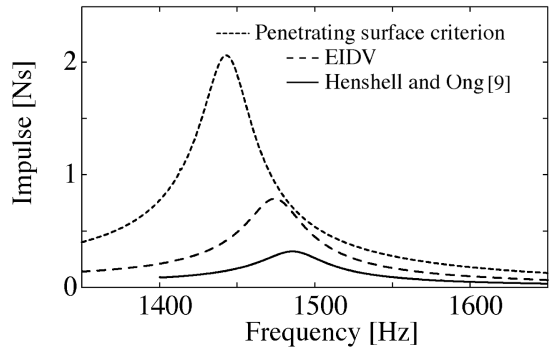

a) $n_{N}=16$ (8 pair)

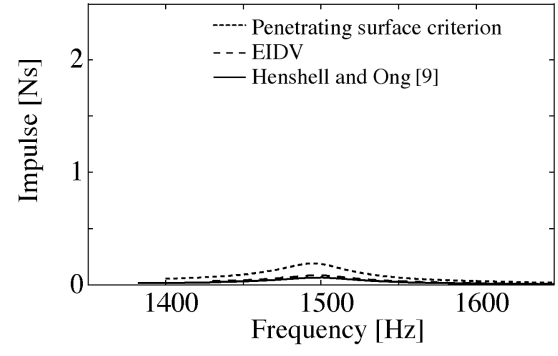

b) $n_{N}=64$ (32 pair)

Fig. 10 Virtual impulse for a period of vibration for NNM 4.

the characteristic area and the characteristic length, $u_{p}$ is the amount of penetration along the surface normals, and $T$ is the period of vibration. The quantity $\hat{F}$ is obtained, based on the calculated time trajectory of displacements of the nodes on the crack surfaces, and it can be thought of as an impulse that does not contribute to the system response. This impulse is not applied to the system when the response is calculated. In other words, the smaller the value of $\hat{F}$, the stricter the BCs are imposed on the nodes over the entirety of the crack surfaces.

First, the forced response analysis was carried out, and the corresponding time history of $u_{p}$ over the entire crack surface was recovered from the vibration response. The integrals in the Eq. (23) were then evaluated by a simple quadrature rule, both in space and time. The metric was calculated for the first and the fourth modes for 8 and 32 pairs chosen by the node sampling methods, and the results are shown in Figs. 9 and 10. As can be seen in Figs. 9 and 10, the virtual impulse varies over the frequency range. In particular, when the frequency of excitation is close to the resonant frequency, the amount of penetration increases as well. However, for all cases, the Henshell and Ong [9] method consistently results in the smallest impulse over the frequency range among the three methods considered. That means that the nonlinear BC on the crack faces is the most strictly enforced by the node patterns chosen by the Henshell and Ong method.

\section{B. Large-Scale Cracked Blade Model}

In this subsection, the performance of the proposed method applied to a FE model of a cracked blade model with a large number of DOFs is demonstrated. A blade model, for which the thickness is $2.5 \times 10^{-3} \mathrm{~m}$ and both chord and span lengths are approximately $5.0 \times 10^{-2} \mathrm{~m}$, was constructed with the Young's modulus of $E=205 \mathrm{GPa}$, density of $\rho=7832 \mathrm{~kg} / \mathrm{m}^{3}$, and Poisson's ratio of $v=0.3$. The blade was discretized with linear and quadratic tetrahedral elements, resulting in a system with 392,361 DOFs, as shown in Fig. 11a. The crack path, as well as its surrounding FE mesh, was generated by a fracture analysis code FRANC3D [44]. The crack surfaces consist of quadratic elements with 487 contact pairs at the edges of vertices of triangles, resulting in 2922 DOFs for both surfaces, as shown in Fig. 11b. The size of the FE model was then reduced down to $2949 \mathrm{DOFs}(0.75 \%$ of the original size) by the primary CMS method, which consists of 2934 physical DOFs (2922 DOFs on crack faces and 12 additional DOFs for forcing) and 15 modal coordinates corresponding to the free-interface normal modes. The proposed method was then applied to the reduced-order model, and the results are shown in Figs. 12 and 13. For the EIDV method, the first 15 modes were used for the calculations. As can be seen in Fig. 12, the EIDV method selects nodes along the rim of the crack faces, similar to the previous case study. In contrast, the

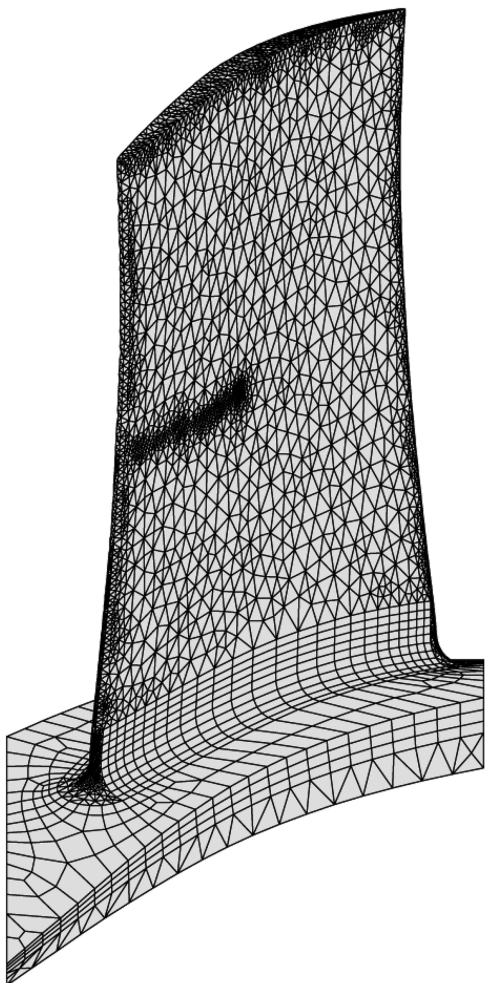

a) FE model

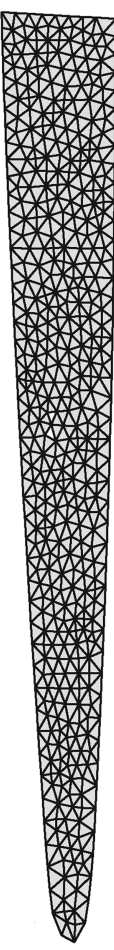

b) Crack surface
Fig. 11 A cracked blade model. 


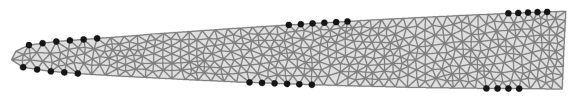

a) $n_{N}=64$ (32 pairs)

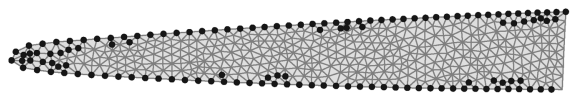

c) $n_{N}=256$ (128 pairs)

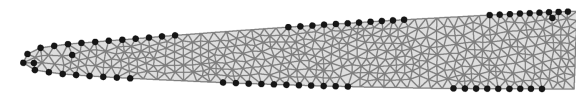

b) $n_{N}=128$ (64 pairs)

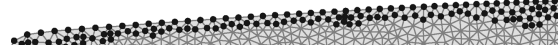

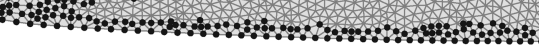

d) $n_{N}=512$ (256 pairs)

Fig. 12 Selected nodes by EIDV method for $n_{\mathcal{B}}=974$ ( 487 pairs).

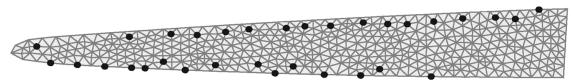

a) $n_{N}=64$ (32 pairs)

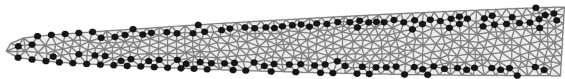

c) $n_{N}=256(128$ pairs $)$

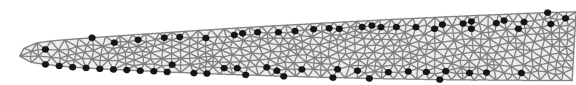

b) $n_{N}=128$ (64 pairs)

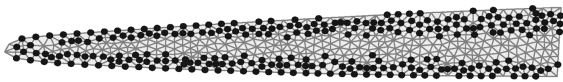

d) $n_{N}=512$ (256 pairs)

Fig. 13 Selected nodes by the Henshell and Ong [9] method for $n_{\mathcal{B}}=974$ (487 pairs).

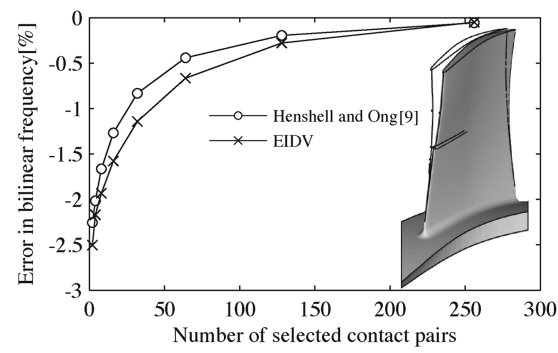

a) NNM 1

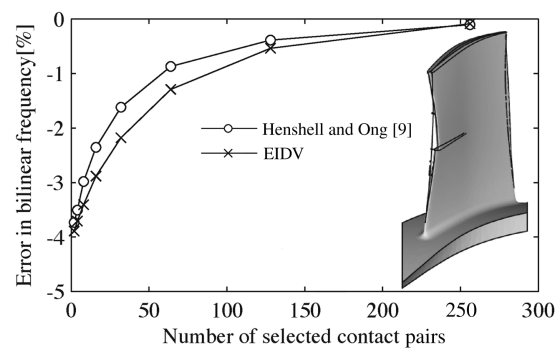

c) NNM 3

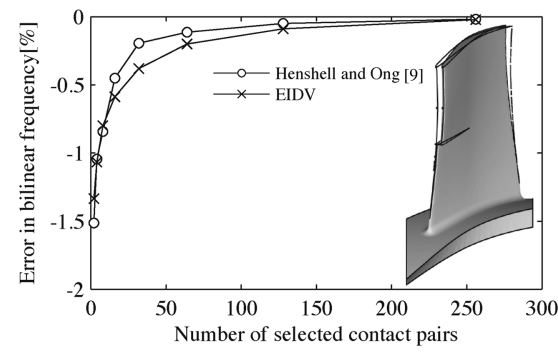

b) NNM 2

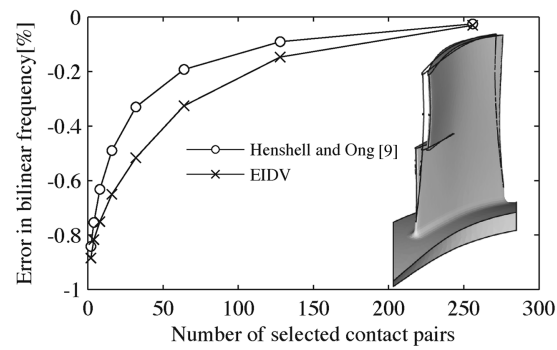

d) NNM 4

Fig. 14 Error in the bilinear frequency for the first four NNMs.

Henshell and Ong [9] method tends to choose the nodes slightly off the crack rim in a more distributed manner over the crack face, as in the previous example. With the selected node patterns, the first four bilinear frequencies were calculated. The first four modes correspond to the first out-of-plane bending, the first torsion, the second out-ofplane bending, and the first chordwise bending mode, respectively. The errors were then calculated, as in the previous example and shown in Fig. 14, along with their linear vibration mode shapes. As can be seen in Fig. 14, the Henshell and Ong method consistently shows better results than the results obtained by the EIDV method.

\section{Conclusions}

In this paper, a novel node sampling methodology for the nonlinear vibration analysis of elastic structures with intermittent contact was proposed. In Sec. III, the modeling framework was developed, based on CMS. The master DOF selection scheme for Guyan reduction [5] was formulated by considering the close relationship between the optimal master DOF selection and the optimal constraint locations for maximizing the fundamental natural frequency. The method is a combination of the sequential elimination method proposed by Henshell and Ong [9] and coordinate transformations to the reduced-order model. Another method for choosing the nodes was also introduced for a comparative study. The alternate method is based on an approach to optimally choose measurement locations, such that the truncated/measured modes become as linearly independent as possible. The method was then applied to a representative FE model in Sec. IV. In Sec. IV.A, the methods were applied to a cracked plate model. Using the selected node patterns, forced response analysis was carried out to evaluate the effects of the selection patterns on the frequency response. Furthermore, the resonant frequencies were calculated by the application of bilinear frequency approximation. It was confirmed that the selected DOFs resulted in accurate predictions of nonlinear resonant frequencies in comparison to the benchmark case of using all DOFs on the crack surfaces. Furthermore, it was demonstrated that the method also achieves a significant reduction in CPU time for the nonlinear forced response calculations, without sacrificing the accuracy in the predicted forced response. Moreover, a method for a posteriori accuracy assessment was introduced by examining the amount of penetration on the crack surfaces during a vibration cycle. In Sec. IV.B, the method was also applied to a cracked blade model 
with a much larger number of DOFs on the crack faces. The node selection patterns, as well as the errors in the bilinear frequencies, conform to the results in the example in Sec. IV.A. For the methods examined, the node patterns selected using the proposed new method consistently showed the best results.

Based on these results, the essential findings and contributions of this paper can be summarized as follows:

1) It was shown mathematically that the selection of the nodes where the nonlinear BCs should be applied for vibration problems with intermittent contact is closely related to the problem of maximizing the fundamental natural frequency of FE models by fixing some of the nodes.

2) The maximization problem can be (approximately) solved by the Henshell and Ong [9] method in conjunction with the CMS method.

3) For simplified yet representative example models, the proposed method produced nodes that give accurate forced response predictions and nonlinear resonant frequencies when the nonlinear BCs are applied on the selected nodes.

4) A posteriori accuracy assessment using numerical examples showed that the forced response analyses with the proposed method retain the largest impulse at the crack surfaces for the frequency ranges of interest

It should also be noted that the proposed method is independent of the geometry of the contact surfaces. That is because the selection procedure is solely dependent on the mass and stiffness of the structure. Therefore, for instance, even if there are distortions of the contact surfaces, the proposed method still works as long as the effects due to the distortions are reflected in the FE mass and stiffness matrices. In contrast, the limitation of the proposed approach is that the geometry of the contact surface has to be known a priori, and the domain of contact surfaces is assumed to remain unchanged during the vibration. That is, if the vibration problems of interest involve dynamic (fast) crack propagation, then the nodes selected by the proposed approach at some time instant might not necessarily be the optimal selection for the entire time duration of interest. However, this is not a problem if the contact problem of interest does not involve fast changes in the contact regions, such as the ones involving gap contact between mechanical components. In addition, local effects on the contact surfaces, such as variations in the grain size on the crack surfaces, were not considered in this paper. These important issues are far beyond the scope of this paper. However, the authors believe that the contributions of this paper shall contribute to further expand the capabilities of model-based vibration analyses involving intermittent contact.

\section{Acknowledgments}

This work was supported in part by Pratt and Whitney, through a subcontract (PW-07-01) to Fracture Analysis Consultants, Inc. and the University of Michigan on a Defense Advanced Research Projects Agency (DARPA)-funded Engine System Prognosis project, and also by a U.S. Air Force Research Laboratory (AFRL) grant. The support of Pratt and Whitney, Fracture Analysis Consultants, Inc., DARPA, and AFRL is gratefully acknowledged. The authors would also like to thank Paul Wawrzynek of Fracture Analysis Consultants for providing the finite-element model of the cracked blade.

\section{References}

[1] Oden, J. T., and Pires, E. B., "Algorithms and Numerical Results for Finite-Element Approximations of Contact Problems with NonClassical Friction Laws," Computers and Structures, Vol. 19, Nos. 1-2, 1984, pp. 137-147. doi:10.1016/0045-7949(84)90212-8.

[2] Simo, J. C., and Laursen, T. A., "An Augmented Lagrangian Treatment of Contact Problems Involving Friction," Computers and Structures, Vol. 42, No. 1, Jan. 1992, pp. 97-116. doi:10.1016/0045-7949(92)90540-G.

[3] Craig, R. R., and Bampton, M. C. C., "Coupling of Substructures for Dynamic Analyses," AIAA Journal, Vol. 6, No. 7, 1968, pp. 1313-1319. doi: $10.2514 / 3.4741$

[4] Nayfeh, A., and Mook, D., Nonlinear Oscillations, Wiley, New York, 1979.

[5] Guyan, R. J., "Reduction of Stiffness and Mass Matrices," AIAA Journal, Vol. 3, No. 2, 1965, p. 380. doi: $10.2514 / 3.2874$

[6] Brahmi, K., Bouhaddi, N., and Fillod, R., "Reduction of Junction Degrees of Freedom Before Assembly in Dynamic Substructuring," Proceedings of the ASME Design Engineering Technical Conference, Vol. 3, 1995, pp. 699-708.

[7] Balmés, E., "Use of Generalized Interface Degrees of Freedom in Component Mode Synthesis," Proceedings of International Modal Analysis Conference, Society for Experimental Mechanics, Bethel, CT, 1996, pp. 204-210.

[8] Castanier, M. P., Tan, Y. C., and Pierre, C., "Characteristic Constraint Modes for Component Mode Synthesis," AIAA Journal, Vol. 39, No. 6, 2001, pp. 1182-1187. doi: $10.2514 / 2.1433$

[9] Henshell, R. D., and Ong, J. H., "Automatic Masters For Eigenvalue Economization," Earthquake Engineering and Structural Dynamics, Vol. 3, No. 4, 1975, pp. 375-383. doi:10.1002/eqe.4290030408

[10] Shah, V. N., and Raymund, M., "Analytical Selection of Masters for the Reduced Eigenvalue Problem," International Journal for Numerical Methods in Engineering, Vol. 18, No. 1, 1982, pp. 89-98. doi:10.1002/nme. 1620180108

[11] Kidder, R. L., "Reduction of Structural Frequency Equations," AIAA Journal, Vol. 11, No. 6, 1973, pp. 892-892. doi: $10.2514 / 3.6852$

[12] Kidder, R. L., "Reply by Author to A. H. Flax," AIAA Journal, Vol. 13, No. 5 , 1975, pp. 702-703. doi: $10.2514 / 3.49798$

[13] Flax, A. H., "Comment on 'Reduction of Structural Frequency Equations'," AIAA Journal, Vol. 13, No. 5, 1975, pp. 701-702. doi: $10.2514 / 3.49797$

[14] Grinenko, N. I., and Mokeev, V. V., "Problems of Studying Vibrations of Structures by the Finite-Element Method," International Applied Mechanics, Vol. 21, No. 3, 1985, pp. 231-235. doi:10.1007/BF00888931

[15] Matta, K. W., "Selection of Degrees of Freedom for Dynamic Analysis," Transactions of the ASME, Series J, Journal of Pressure Vessel Technology, Vol. 109, No. 1, 1987, pp. 65-69. doi:10.1115/1.3264857

[16] Bouhaddi, N., and Fillod, R., "A Method for Selecting Master DOF in Dynamic Substructuring Using the Guyan Condensation Method," Computers and Structures, Vol. 45, Nos. 5-6, 1992, pp. 941-946. doi:10.1016/0045-7949(92)90052-2

[17] Hitziger, T., Mackens, W., and Voss, H., "A Condensation-Projection Method for Generalized Eigenvalue Problems," High Performance Computing 1, edited by H. Power, and C. A. Brebbia, Elsevier, London, 1995, pp. 239-282.

[18] Kim, K. O., and Choi, Y. J., "Energy Method for Selection of Degrees of Freedom in Condensation," AIAA Journal, Vol. 38, No. 7, 2000, pp. 1253-1259. doi:10.2514/2.1095

[19] Cho, M., and Kim, H., "Element-Based Node Selection Method for Reduction of Eigenvalue Problems," AIAA Journal, Vol. 42, No. 8, 2004, pp. 1677-1684. doi: $10.2514 / 1.5407$

[20] Kim, H., and Cho, M., "Two-level Scheme for Selection of Primary Degrees of Freedom and Semi-Analytic Sensitivity Based on the Reduced System," Computer Methods in Applied Mechanics and Engineering, Vol. 195, Nos. 33-36, 2006, pp. 4244-4268. doi:10.1016/j.cma.2005.08.004

[21] O'Callahan, J., "A Procedure for an Improved Reduced System (IRS) Model," Proceedings of the 7th International Modal Analysis Conference, Society for Experimental Mechanics, Inc., Bethel, CT, Jan. 1989, pp. 17-21.

[22] Li, W., "A Degree Selection Method of Matrix Condensations for Eigenvalue Problems," Journal of Sound and Vibration, Vol. 259, No. 2, 2003, pp. 409-425. doi:10.1006/jsvi.2002.5336

[23] Oh, D., and Park, Y., "Order Reduction Based on Singular Values of a Modal matrix," Mechanical Systems and Signal Processing, Vol. 8, No. 1, 1994, pp. 63-79. doi:10.1006/mssp. 1994.1005

[24] Udwadia, F. E., "Methodology for Optimum Sensor Locations for Parameter Identification in Dynamic Systems," Journal of Engineering Mechanics, Vol. 120, No. 2, 1994, pp. 368-390. 
doi:10.1061/(ASCE)0733-9399(1994)120:2(368)

[25] Penny, J. E. T., Friswell, M. I., and Garvey, S. D., "Automatic Choice of Measurement Locations for Dynamic Testing," AIAA Journal, Vol. 32, No. 2, 1994, pp. 407-414. doi: $10.2514 / 3.11998$

[26] Kammer, D. C., "Sensor Placement for On-Orbit Modal Identification and Correlation of Large Space Structures," Journal of Guidance, Control, and Dynamics, Vol. 14, No. 2, 1991, pp. 251-259. doi: $10.2514 / 3.20635$

[27] Zhu, J., and Zhang, W., "Maximization of Structural Natural Frequency with Optimal Support Layout," Structural and Multidisciplinary Optimization, Vol. 31, No. 6, 2006, pp. 462-469. doi:10.1007/s00158-005-0593-2

[28] Åkesson, B., and Olhoff, N., "Minimum Stiffness of Optimally Located Supports for Maximum Value of Beam Eigenfrequencies," Journal of Sound and Vibration, Vol. 120, No. 3, 1988, pp. 457-463. doi:10.1016/S0022-460X(88)80218-9

[29] Szelag, D., and Mroz, Z., "Optimal Design of Vibrating Beams with Unspecified Support Reactions," Computer Methods in Applied Mechanics and Engineering, Vol. 19, No. 3, 1979, pp. 333-349. doi:10.1016/0045-7825(79)90063-X

[30] Won, K. M., and Park, Y. S., "Optimal Support Positions for a Structure to Maximize its Fundamental Natural Frequency," Journal of Sound and Vibration, Vol. 213, No. 5, 1998, pp. 801-812. doi:10.1006/jsvi.1997.1493

[31] Butcher, E., and Lu, R., "Order Reduction of Structural Dynamic Systems with Static Piecewise Linear Nonlinearities," Nonlinear Dynamics, Vol. 49, No. 3, 2007, pp. 375-399. doi:10.1007/s11071-006-9129-6

[32] O'Callahan, J., Avitabile, P., and Riemer, R., "System Equivalent Reduction Expansion Process (SEREP)," Proceedings of the 7th International Modal Analysis Conference, Society for Experimental Mechanics, Inc., Bethel, CT, Jan. 1989, pp. 29-37.

[33] Friswell, M. I., Garvey, S. D., and Penny, J. E. T., "Model Reduction Using Dynamic and Iterated IRS Techniques," Journal of Sound and Vibration, Vol. 186, No. 2, 1995, pp. 311-323. doi:10.1006/jsvi.1995.0451

[34] Friswell, M. I., Garvey, S. D., and Penny, J. E. T., "The Convergence of the Iterated IRS Method," Journal of Sound and Vibration, Vol. 211, No. 1, 1998, pp. 123-132. doi:10.1006/jsvi.1997.1368

[35] Friswell, M. I., Penny, J. E. T., and Garvey, S. D., "Using Linear Model Reduction to Investigate the Dynamics of Structures with Local NonLinearities," Mechanical Systems and Signal Processing, Vol. 9, No. 3, 1995, pp. 317-328. doi:10.1006/mssp. 1995.0026

[36] Saito, A., Castanier, M. P., Pierre, C., and Poudou, O., "Efficient Nonlinear Vibration Analysis of the Forced Response of Rotating Cracked Blades," Journal of Computational and Nonlinear Dynamics, Vol. 4, No. 1, 2009, Paper 011005. doi: $10.1115 / 1.3007908$

[37] Hintz, R. M., "Analytical Methods in Component Modal Synthesis," AIAA Journal, Vol. 13, No. 8, 1975, pp. 1007-1016. doi: $10.2514 / 3.60498$

[38] Herting, D. N., "A General Purpose, Multi-Stage, Component Modal Synthesis Method," Finite Elements in Analysis and Design, Vol. 1, No. 2, 1985, pp. 153-164. doi:10.1016/0168-874X(85)90025-3

[39] Poudou, O., and Pierre, C., "Hybrid Frequency-Time Domain Methods for the Analysis of Complex Structural Systems with Dry Friction Damping," 44th AIAA/ASME/ASCE/AHS/ASC Structures, Structural Dynamics, and Materials Conference, AIAA Paper 2003-1411, April 2003.

[40] Saito, A., Castanier, M., and Pierre, C., "Estimation and Veering Analysis of Nonlinear Resonant Frequencies of Cracked Plates," Journal of Sound and Vibration, Vol. 326, Nos. 3-5, 2009, pp. 725739. doi:10.1016/j.jsv.2009.05.009

[41] Voss, H., "An Error Bound for Eigenvalue Analysis by Nodal Condensation," Numerical Treatment of Eigenvalue Problems, edited by J. Albrecht, L. Collatz, and W. Velete, Vol. 3, Birkhäuser-Verlag, Stuttgart, Germany, 1983, pp. 205-214.

[42] Thomas, D. L., "Errors in Natural Frequency Calculations using Eigenvalue Economization," International Journal for Numerical Methods in Engineering, Vol. 18, No. 10, 1982, pp. 1521-1527. doi:10.1002/nme.1620181007

[43] Mackens, W., and Voss, H., "Nonnodal Condensation of Eigenvalue Problems," Zeitschrift für Angewandte Mathematik und Mechanik, Vol. 79, No. 4, 1999, pp. 243-255. doi:10.1002/(SICI)1521-4001(199904)79:4<243::AID-ZAMM243> 3.0. $\mathrm{CO} ; 2-2$

[44] Carter, B. J., Wawrzynek, P. A., and Ingraffea, A. R., "Automated 3-D Crack Growth Simulation," International Journal for Numerical Methods in Engineering, Vol. 47, No. 1-3, 2000, pp. 229-253. doi:10.1002/(SICI)1097-0207(20000110/30)47:1/3<229::AID-NME769> 3.0. $\overline{\mathrm{CO} ; 2-2}$
R. Kapania Associate Editor 